\title{
Cation dynamics and structural stabilization in formamidinium lead iodide perovskites
}

\author{
Kacper Drużbicki, ${ }^{1,2, *}$ Rasmus Lavén, ${ }^{3}$ Jeff Armstrong, ${ }^{4}$ Lorenzo \\ Malavasi, ${ }^{5}$ Felix Fernandez-Alonso, ${ }^{2,6,7,8}$ and Maths Karlsson ${ }^{3}$ \\ ${ }^{1}$ Polish Academy of Sciences, Centre of Molecular and Macromolecular Studies, Sienkiewicza 112, 90-363 Lodz, Poland \\ ${ }^{2}$ Materials Physics Center, CSIC-UPV/EHU, Paseo Manuel de Lardizabal 5, 20018 Donostia - San Sebastian, Spain \\ ${ }^{3}$ Department of Chemistry and Chemical Engineering, \\ Chalmers University of Technology, SE-412 96 Göteborg, Sweden \\ ${ }^{4}$ ISIS Facility, Rutherford Appleton Laboratory, Harwell Oxford, Didcot, Oxfordshire OX11 0QX, U. K. \\ ${ }^{5}$ Department of Chemistry and INSTM, University of Pavia, Viale Taramelli 16, Pavia 27100, Italy \\ ${ }^{6}$ IKERBASQUE, Basque Foundation for Science, Plaza Euskadi 5, 48009 Bilbao, Spain \\ ${ }^{7}$ Donostia International Physics Center (DIPC), \\ Paseo Manuel de Lardizabal 4, 20018 Donostia - San Sebastian, Spain \\ ${ }^{8}$ Department of Physics and Astronomy, University College London, Gower Street, London, WC1E 6BT, U. K.
}

\begin{abstract}
The vibrational dynamics of pure and methylammonium-doped formamidinium lead iodide perovskites $\left(\mathrm{FAPbI}_{3}\right)$ has been investigated by high-resolution neutron spectroscopy. For the first time, we provide an exhaustive and accurate analysis of the cation vibrations and underlying local structure around the organic moiety in these materials using first-principles electronic-structure calculations validated by the neutron data. Inelastic Neutron Scattering experiments on $\mathrm{FAPbI}_{3}$ provide direct evidence of the formation of a low-temperature orientational glass, unveiling the physico-chemical origin of phase metastability in the tetragonal structure. Further analysis of these data provides a suitable starting point to understand and explore the stabilization of the perovskite framework via doping with small amounts of organic cations. In particular, we find that hydrogen bonds in $\mathrm{FAPbI}_{3}$ are strengthened in a synergistic manner as a result of cage deformation induced by the dynamics of the neighbouring methylammonium cations.
\end{abstract}

\section{INTRODUCTION}

Organometal Halide Perovskites (OHP's) have recently attracted an enormous interest due to their promising applications as absorbers in photovoltaic devices. 1 The prototypical OHP is methylammonium lead iodide $\left(\mathrm{CH}_{3} \mathrm{NH}_{3} \mathrm{PbI}_{3}\right.$, or $\mathrm{MAPbI}_{3}$ for short $)$. The main disadvantage of $\mathrm{MAPbI}_{3}$ is, however, its poor long-term stability towards humidity and heat. $2 \sqrt{5}$ Formamidinium lead iodide ( $\mathrm{HC}\left[\mathrm{NH}_{2}\right]_{2} \mathrm{PbI}_{3}$, or $\mathrm{FAPbI}$ for short) is currently attracting a lot of attention because it shows a much higher environmental stability, and its cubic phase has a close to ideal bandgap for photoabsorption (1.47 eV). 6, 7. However, FAPbI $_{3}$ shows a complex polymorphism behaviour which is currently considered to be a prime factor limiting its application potential. 8

The structural picture of both $\mathrm{MAPbI}_{3}$ and $\mathrm{FAPbI}_{3}$ remains elusive to a great extent, since its better understanding is hindered by the presence of both static and dynamical disorder, as well as by the formation of domain structures of multiple origins. These idiosyncrasies have been highlighted, for instance, in the total-scattering experiments reported by Malavasi and co-workers, bringing to the fore the relevance and importance of ubiquitous short-range octahedral distortions in OHP's. 9 11]

The phase diagrams of both systems under interest and their mixtures is depicted in Fig. 1. In principle, it is commonly accepted that $\mathrm{MAPbI}_{3}$ shows three

* E-mail: kacper.druzbicki@ehu.eus Tel: +34943015453 different crystal structures depending on the temperature, i.e. the low-temperature orthorhombic phase for $T<160 \mathrm{~K}$; the tetragonal phase for $160 \mathrm{~K}<T<$ $330 \mathrm{~K}$; and the high-temperature cubic phase for $T>$ $330 \mathrm{~K}$. 12 The space group assignment of each phase of $\mathrm{MAPbI}_{3}$ has been, however, debatable from the very beginning. Poglitsch and Weber solved the orthorhombic structure in the non-centrosymmetric space group $P n a 2_{1}$, 13] which has been eventually refined as the Pnma structure by Baikie et al. [14] Using extensive $a b$ initio modelling combined with inelastic neutron scattering (INS) experiments, Drużbicki et al. 15 showed that the Pnma model is insufficient to describe the experimental data, pointing at the importance of octahedral deformations. Furthermore, with low-temperature X-ray diffraction, Baikie et al. [14] revealed the presence of some noticeable residual electron density at distances unreasonably close to the iodine positions, which leaves their space group assignment questionable. Similarly, the presence of high-residual electron density peaks was detected by Breternitz et al. [16] in the high-resolution Xray study of the tetragonal phase. However, the commonly accepted centrosymmetric $14 / \mathrm{mcm}$ model of the room-temperature phase has been recently questioned, following the discovery of ferroelectric behavior. [17] This has been further resolved by Breternitz et al. with the refinement of the structure within $I 4 \mathrm{~cm}$, which is the only polar maximal subgroup of $I 4 / \mathrm{mcm}$. The average structure of the high-temperature cubic phase has been described with the $P m \overline{3} m$ space group. However, recent high-resolution X-ray total-scattering studies postulate the system to be made up of small dynamic tetragonal 


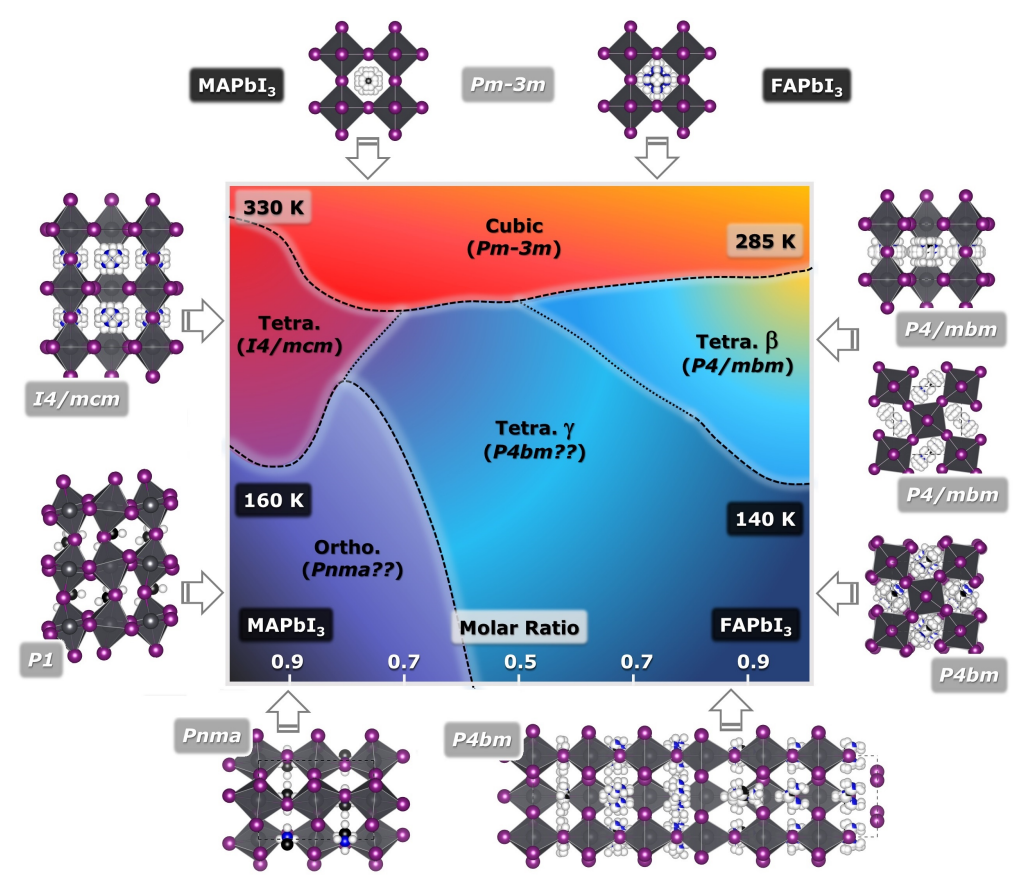

FIG. 1: The phase diagram of the perovskite solid solutions $\mathrm{MA}_{1-\mathrm{x}} \mathrm{FA}_{\mathrm{x}} \mathrm{PbI}_{3}$ across the temperature range of $10-365 \mathrm{~K}$, according to Francisco-López et al. 18 Note that the mixtures studied in the present work correspond to the solutions $\mathrm{MA}_{0.1} \mathrm{FA}_{0.9} \mathrm{PbI}_{3}$ and $\mathrm{MA}_{0.4} \mathrm{FA}_{0.6} \mathrm{PbI}_{3}$, respectively. The phase transition temperatures marked on the diagram refer to pure parent components. Note that the low-temperature structures are questionable (the space groups denoted with the question marks). For each phase considered (see the arrows), the corresponding crystal structures of pure $\mathrm{MAPbI}_{3}$ and $\mathrm{FAPbI}_{3}$ are presented as insets, with selected geometrical projections. Distortions of the $\mathrm{PbI}_{6}$ octahedra are the characteristic features of the alternative $P 1$ model of the low-temperature structure of $\mathrm{MAPbI}_{3}$. 15] The most-reliable model of the low-temperature $\gamma$-polymorph of $\mathrm{FAPbI}_{3}$ published to date is of $P 4 \mathrm{bm}$ symmetry. [19] This model is based on a $1 \times 1 \times 6$ expansion of the $\beta$-phase model $(P 4 / \mathrm{mbm})$, where the $\mathrm{FA}^{+}$cation is rotated incrementally around the long cell axis.

domains at $350 \mathrm{~K}$. 20, 21 Moreover, these domains were found to be left over from structural phase transitions that take place tens to hundreds of degrees below. 21 These considerations are in line with the conflicting evidence from various experimental and theoretical treatments of the degree of ordering of the organic cation in the orthorhombic phase. 22]

In the above context, the atomic structure of $\mathrm{FAPbI}_{3}$ is far less understood. At room temperature (RT), $\mathrm{FAPbI}_{3}$ has a stable hexagonal non-perovskite phase (yellow $\delta$ polymorph, $P 6_{3} m c$ ). 7, 23. However, a metastable cubic perovskite phase (black $\alpha$-phase, $P m \overline{3} m$ ), with an optimal band gap for photovoltaics, can be stabilized at RT for hours to weeks (depending on sample storage etc.) via heating above the hexagonal-to-cubic phase transition occuring around $410 \mathrm{~K}$. 7, 8, 23, Upon cooling from the cubic phase, a tetragonal phase $(\beta$-phase, $P 4 / \mathrm{mbm}$ ) is formed below $285 \mathrm{~K}$, and yet another structure is found below $140 \mathrm{~K}$ ( $\gamma$-phase). [19, 24, 28, The structure of the low-temperature $\gamma$-polymorph is still under debate. Based on powder X-ray diffraction (PXRD), Fabini et al. [25] refined the $\gamma$-phase in a new primitive tetragonal unit cell retaining $P 4 / \mathrm{mbm}$ symmetry, whilst Chen et al. [19] simultaneously refined the neutron and X-ray diffraction patterns in a $1 \times 1 \times 6$ super- cell $(P 4 b m)$ of the intermediate tetragonal phase, with the $\mathrm{FA}^{+}$cations rotated in increments around the $c$-axis. More recently, neutron powder diffraction experiments by Weber et al. 28] pointed towards a locally disordered $\gamma$-phase with possibly no long-range ordering of the $\mathrm{FA}^{+}$ organic cations. The formation of an orientational glass at low temperatures has also been reported in previous studies. 22, 29] Therefore, a complete understanding of the low-temperature phase of $\mathrm{FAPbI}_{3}$ remains elusive.

Due to aforementioned lack of phase stability in $\mathrm{FAPbI}_{3}$, considerable efforts have been focused on the study of the mixed-cation systems $\mathrm{MA}_{1-\mathrm{x}} \mathrm{FA}_{\mathrm{x}} \mathrm{PbI}_{3}$, as they have been shown to stabilize the cubic perovskite structure of $\mathrm{FAPbI}_{3}$ at RT even for relatively low concentrations of the MA dopant. 7] Additionally, these mixed systems show a higher stability towards humidity and heat than pure $\mathrm{MAPbI}_{3}$, and are currently among the materials with the highest photovoltaic efficiency. 3033 Combining Raman scattering and photoluminescence (PL) spectroscopy, Francisco-López et $a l$. have recently reported the compositional phase diagram of $\mathrm{MA}_{1-\mathrm{x}} \mathrm{FA}_{\mathrm{x}} \mathrm{PbI}_{3}$ solid solutions across the temperature range $10-365 \mathrm{~K}$. 18]

Notwithstanding these recent developments, many fundamental aspects of $\mathrm{FAPbI}_{3}$ remain poorly understood. 

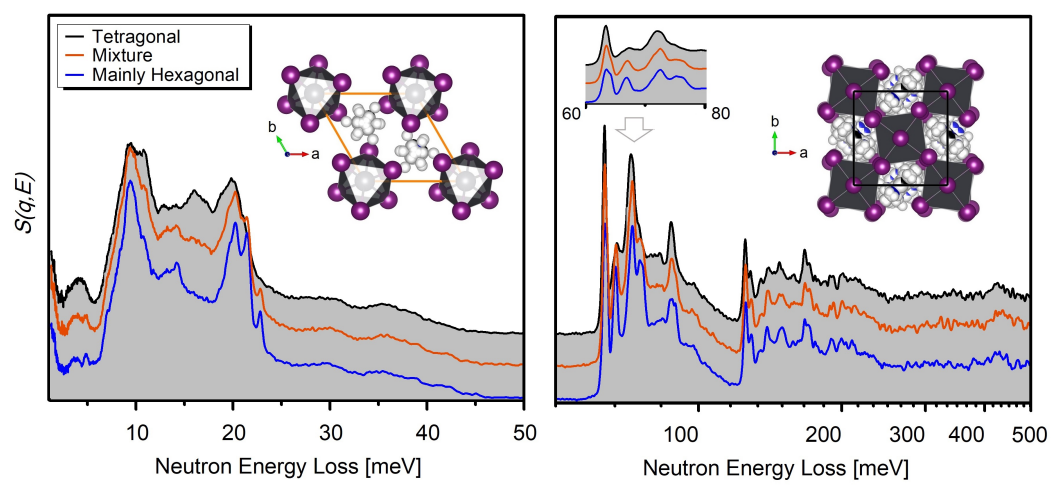

FIG. 2: INS spectra measured on $(i)$ starting material of $\mathrm{FAPbI}_{3}$ (orange curve; mixture of both perovskite and non-perovskite phases); ( $i$ i) after storage in vacuo for a period of three-months (blue curve; increased amount of the hexagonal phase); and (iii) after annealing at $165^{\circ} \mathrm{C}$ (black shaded curve; tetragonal phase). For ease of comparison, the spectra have been normalized and vertically shifted, and the spectral range of $60-80 \mathrm{meV}$ is given as an inset. The atomic projections show the crystallographic structures of the low-temperature hexagonal (left) and tetragonal (right) phases according to Chen et al. [19.

In particular, we note important gaps in our knowledge concerning the low-temperature structure of $\mathrm{FAPbI}_{3}$, which is believed to form an orientational glass, or how doping with small amounts of $\mathrm{MA}^{+}$cations facilitates the stabilization of the perovskite framework of $\mathrm{FAPbI}_{3}$. In addition, as compared to $\mathrm{MAPbI}_{3}$, relatively little is known about the dynamics of the $\mathrm{FA}^{+}$cation which, however, is believed to be more of an intricate nature. 34]

To date, $\mathrm{FAPbI}_{3}$ has been primarily studied by diffraction techniques, [19, 24, 25, 27, 28, thereby providing information on the time-averaged structure. On the contrary, the use of spectroscopic techniques allows access to atomic and molecular correlation functions in both space and time and, thus, provide additional insights into the local structure and ensuing dynamics. Relative to other techniques, Inelastic Neutron Scattering (INS) is, in particular, superbly sensitive to the organic cation in OHP's, whose dynamics largely determines charge localization in these materials. 35. So far, the study of atomic motions in $\mathrm{FAPbI}_{3}$ using INS spectroscopy has been primarily focused on the inorganic components $\mathrm{Pb}$ and $\mathrm{I}$. This work has evinced the extreme softness of the Pb-I framework, leaving the hydrogen dynamics quite unexplored and limited to a narrow energy transfer range (0$15 \mathrm{meV}$ ). 36, 37. Further insights have been provided for the analogous $\mathrm{FAPbBr}_{3}$ system, as recently reported by Mozur et al. 38.

As in the previous case of $\mathrm{MAPbI}_{3}$ [15, we take the ensuing dynamics of the organic cation as a sensitive probe of local structure. In this work, the INS spectrum of $\mathrm{FAPbI}_{3}$ is presented and extensively discussed for the first time. We focus on the low-temperature tetragonal structure observed upon cooling from the metastable cubic polymorph at RT. Ab initio modelling using timedependent and time-independent methodologies provide an accurate description of the measured INS response of $\mathrm{FAPbI}_{3}$. We extend this analysis onto the $\mathrm{MA}^{+}-$doped
$\mathrm{FAPbI}_{3}$ mixtures, in order to provide new insights into the stabilisation of these solid solutions.

\section{RESULTS AND DISCUSSION}

It is known that $\mathrm{FAPbI}_{3}$ decomposes into a nonperovskite hexagonal phase ( $\delta$-polymorph), which is the thermodynamically stable form at RT. [19, 28, 39] Our starting material is a physical mixture of the metastable perovskite (room-temperature cubic $\alpha$-phase) and the stable non-perovskite $\delta$-polymorph [19, 28]. Taking this knowledge into account, a first INS measurement was performed by cooling our starting material to base temperature $(10 \mathrm{~K})$, where the contributing polymorphs are transformed into their respective low-temperature phases - namely, the low-temperature perovskite $\gamma$-phase as well as the low-temperature hexagonal phase, hereafter denoted LT-Hex. [19] The experiment was repeated after three months, in order to increase the relative amount of the non-perovskite fraction. The perovskite structure of $\mathrm{FAPbI}_{3}$ can be, however, restored by heating the sample above the hexagonal-to-cubic phase transition occurring around $400 \mathrm{~K}$. 8, 24, 28, A third INS measurement was, hence, performed immediately after a heat treatment at $438 \mathrm{~K}$ for about 10 minutes. [40] The sample was then quenched down to base temperature in order to observe the pure $(\gamma$-phase $)$ of $\mathrm{FAPbI}_{3}$. [19] These INS data are presented in Fig. 2.

As shown in Fig. 2, thermal annealing results in rather subtle changes in spectral intensities, and in the disappearance of some shoulders. Most importantly, we note a downward shift of the intense feature at ca. $20 \mathrm{meV}$ and the loss of its fine structure, as well as a clear growth in the intensity of the band at $15 \mathrm{meV}$. From an analysis of close contacts in both polymorphs (see the insets to Fig. 22), this effect appears to be related to the weakening of 
specific interactions, e.g., hydrogen-bonds, due to the enhanced disorder of the $\mathrm{FA}^{+}$units in the tetragonal phase. The range above $50 \mathrm{meV}$ is hardly sensitive to the underlying phase, only resulting in relatively minor frequency shifts and intensity modulations, which can be further linked to an increased cation disorder in the perovskite phase affecting the hydrogen-bond network.

At this juncture, we note that the resulting INS spectrum may depend on the way the dynamic structure factor $S(q, E)$ is probed via INS. These considerations are corroborated through a comparison of INS data for single-crystal and powder samples of an analogous $\mathrm{FAPbBr}_{3}$ system, obtained with triple-axis and indirectgeometry neutron spectrometers, respectively. 37, 38. The overall intensity progression of the INS spectrum of $\mathrm{FAPbI}_{3}$ presented in this work is very similar to that observed by Mozur et al. on powder $\mathrm{FAPbBr}_{3}$, collected on the VISION spectrometer (SNS, Oak Ridge). 38]

Figure 3 shows the INS spectra of the parent materials, along with the $\mathrm{MA}_{1-\mathrm{x}} \mathrm{FA}_{\mathrm{x}} \mathrm{PbI}_{3}$ mixtures of relevance to this work. In order to bring to the fore the relative contributions of either $\mathrm{MAPbI}_{3}$ and $\mathrm{FAPbI}_{3}$ to total intensities, these data have been normalized to the height of those spectral bands that are least affected by cation mixing. Using this normalization procedure, difference spectra $\left(\mathrm{MA}_{1-\mathrm{x}} \mathrm{FA}_{\mathrm{x}} \mathrm{PbI}_{3}-\mathrm{FAPbI}_{3}\right)$ were calculated to highlight $\mathrm{MAPbI}_{3}$ contributions to the total intensity (see the red curves in Fig. 3).

The INS spectrum of $\mathrm{MAPbI}_{3}$ shows several sharp peaks in the region below $40 \mathrm{meV}$. The vibrational dynamics of pure $\mathrm{MAPbI}_{3}$ has already been studied extensively in a previous INS study. [15] Here, we emphasize the recent upgrade of the TOSCA spectrometer, [41 45], which has allowed us to explore the terahertz range with a much higher signal-to-noise ratio relative to previous work. 15] The sharp modes below $30 \mathrm{meV}$ are governed by combined $\mathrm{MA}^{+}$librations and vibrations of the leadiodide framework. 15] The peaks above $30 \mathrm{meV}$ mainly consist of internal molecular vibrations. In particular, the sharp and intense peak at $\sim 37 \mathrm{meV}$ has been assigned to a torsional mode of MA and was found to be particularly sensitive to the local crystal environment of the $\mathrm{MA}^{+}$cation. 15.

Compared to $\mathrm{MAPbI}_{3}$, the INS spectrum of $\mathrm{FAPbI}_{3}$ exhibits broader features in the low-energy region of $\sim 6-30 \mathrm{meV}$, corresponding to molecular librations combined with inorganic lattice deformations. These differences indicate that the orientation of the $\mathrm{FA}^{+}$cations are more disordered at $10 \mathrm{~K}$, as compared to $\mathrm{MA}^{+}$in $\mathrm{MAPbI}_{3}$, where the cations arrange in highly preferable directions. 12 This observation is in agreement with previous studies by neutron diffraction, 28, thermophysical measurements, 22, and nuclear magnetic resonance, 29. which have indicated the formation of an $\mathrm{FAPbI}_{3}$ orientational glass at temperatures below $100 \mathrm{~K}$.

For the mixed stoichiometries, the INS features shown in Fig. 3 are also very similar to those of tetragonal $\mathrm{FAPbI}_{3}$, consistent with that both of the mixed stoi-

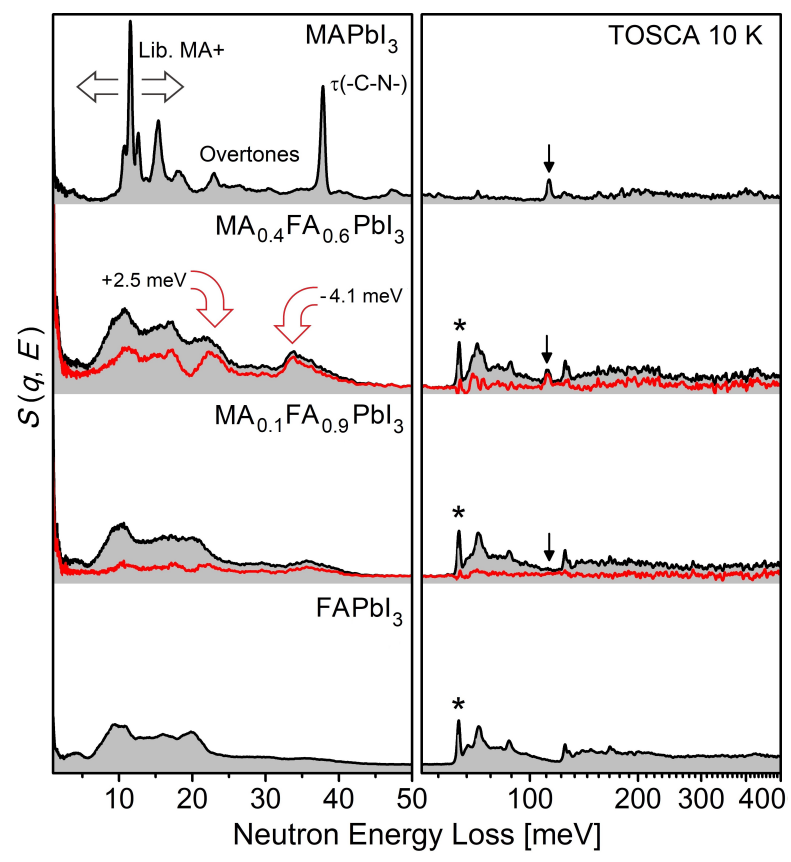

FIG. 3: INS spectra of $\mathrm{FAPbI}_{3}$ and $\mathrm{MAPbI}_{3}$ along with their mixtures, recorded on TOSCA at $10 \mathrm{~K}$. The spectra of the mixtures were normalized w.r.t. the one for pure $\mathrm{FAPbI}_{3}$ using the intensity of the band marked with the asterisk. The spectrum of pure $\mathrm{MAPbI}_{3}$ was normalized w.r.t. the spectrum of $\mathrm{MA}_{0.4} \mathrm{FA}_{0.6} \mathrm{PbI}_{3}$, using the band marked with an arrow. Consequently, the difference spectra for the mixtures (the red curves) were calculated as $\mathrm{MA}_{1-\mathrm{x}} \mathrm{FA}_{\mathrm{x}} \mathrm{PbI}_{3}$ - $\mathrm{FAPbI}_{3}$. The most prominent spectral shifts upon sample mixing are marked with red arrows. The same intensity scale has been used for all the spectra presented in this figure.

chiometries have the same low-temperature structure as $\mathrm{FAPbI}_{3}$.46] The difference spectra, which are mainly highlighting the $\mathrm{MA}^{+}$features, reflect a strong damping of intensity in the low-frequency part of the spectrum. Noticeably, there is a progressive upward shift of the spectral peak at ca. $20 \mathrm{meV}$ with increasing $\mathrm{MAPbI}_{3}$ content (by ca. $2.5 \mathrm{meV}$ ), which, however cannot be unequivocally assigned at this stage. Also, the peak observed at ca. $37 \mathrm{meV}$ in pure $\mathrm{MAPbI}_{3}$, assigned to a disrotary mode of the methyl and/or ammonia groups of $\mathrm{MAPbI}_{3}$ (denoted as $\tau(-\mathrm{C}-\mathrm{N}-)$ ), 15 is substantially modified in these mixtures (by ca. $4 \mathrm{meV}$ ). A similar shift and damping have been observed by Mozur et al. [4] for the case of $\mathrm{MA}_{1-\mathrm{x}} \mathrm{Cs}_{\mathrm{x}} \mathrm{PbBr}_{3}$, where the formation of an orientational $\mathrm{MA}^{+}$glass was observed at low temperature for relatively low concentrations of the $\mathrm{Cs}^{+}$cation. Similarly, the most intense peak observed within the librational part of the spectrum (see Lib. $\mathrm{MA}^{+}$) at ca. 10 $\mathrm{meV}$ for crystalline $\mathrm{MAPbI}_{3}$ can be assigned to the mode arising from a reorientation of the whole $\mathrm{MA}^{+}$molecule about the long molecular axis. These molecular motions are modulated by well-defined hydrogen-bonding interactions between the organic cation and the inorganic lattice, giving rise to sharp spectral features in crystalline 
$\mathrm{MAPbI}_{3}$. From these considerations, we may conclude that $\mathrm{MA}^{+}$in the mixtures experiences a largely disordered local environment, with little evidence to support the idea of long-range order for either $\mathrm{FA}^{+}$or $\mathrm{MA}^{+}$.

To gain further and much-needed quantitative insights into the INS data, we have performed first-principles calculations. To this end, we have explored several computational models:

(i) The first one (hereafter, $\beta$-model) takes as starting point the structure of the $\mathrm{FAPbI}_{3}$ tetragonal cell provided by Fabini et al. 25] This structure has been solved in the $P 4 / \mathrm{mbm}$ space group, with the $\mathrm{FA}^{+}$cation represented as a point mass. Since the cation is thought to be dynamically disordered between preferred orientations, we have considered different scenarios by transforming the initial $P 4 / \mathrm{mbm}$ structure into a number of alternative lower-symmetry configurations. As further detailed in the SI, this approach had limited success in describing the experimental INS spectrum of the low-temperature phase of $\mathrm{FAPbI}_{3}$. These calculations indicate that the total number of cations contained in the $\beta$-model per crystallographic cell is insufficient to account for all spectral features.

(ii) Based on the above results for the $\beta$-model, an extended model of the disordered low-temperature phase was used as initial input (hereafter $\gamma$-model, $P 4 b m$ ). This structure has been proposed by Chen et al. [19, 26, based on powder neutron diffraction experiments, and its reliability has been recently confirmed by Webber et al. 28 The cell constants were fixed to the values measured at $15 \mathrm{~K}(a=8.87740 \AA, c=37.67240 \AA)$, [19, 26] whereas the $\mathrm{FA}^{+}$cations $(\times 12$ ions) were extracted from the crystallographic model and oriented randomly, to account for molecular disorder. This structure (with a resulting $P 1$ ) symmetry) was found to be sufficient to account for all INS features and, therefore, it has been used for subsequent normal-mode analysis using Harmonic Lattice Dynamics (HLD). (iii) Finally, we have performed Ab Initio Molecular Dynamics (AIMD) simulations on an extended $\gamma$-model of $\mathrm{FAPbI}_{3}$ consisting of a larger $2 \times 2 \times 1$ supercell containing $48 \mathrm{FA}^{+}$ions and with lattice parameters $a=b=17.75480 \AA$ and $c=$ $37.67240 \AA$. This model is compared with AIMD simulations on $\mathrm{MAPbI}_{3}$ for a $2 \times 2 \times 2$ supercell containing $32 \mathrm{MA}^{+}$cations. This $\mathrm{MAPbI}_{3}$ supercell uses the $P 1$ model $(Z=4)$ of the low-temperature phase from previous work. [15] The primitive model used for reference HLD calculations of $\mathrm{MAPbI}_{3}$ is described by the highest possible symmetry corresponding to the $C c$ space group $(Z=2)$.

The above computational models adopted throughout this work are summarised in Fig. 4, and more details are relegated to the SI. These can be used to provide a quantitative interpretation of the INS spectrum of $\mathrm{FAPbI}_{3}$, which has been summarized in Table 1 . The spectra resulting from both HLD and AIMD simulations are displayed in Fig. 5. For consistency, we confront these outputs with the theoretical calculations performed for

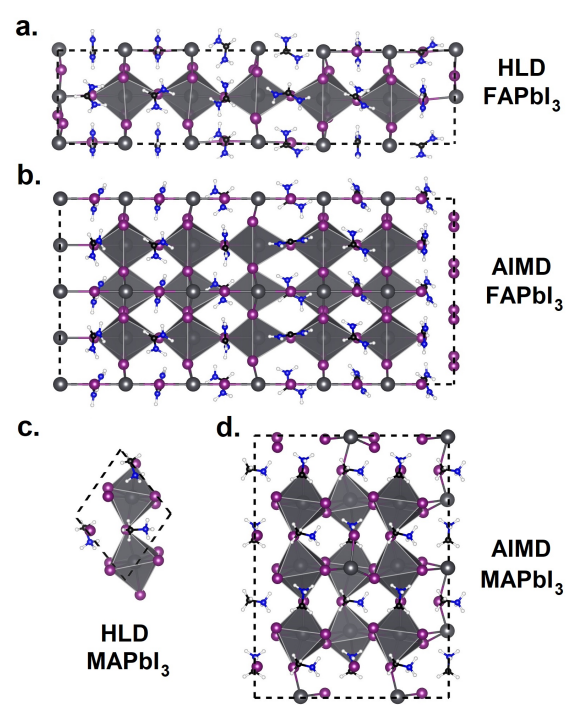

FIG. 4: The structural models of $\mathrm{FAPbI}_{3}$ (a. - b.) and $\mathrm{MAPbI}_{3}$ (c. - d.) adopted in the ab initio modelling detailed in the main text. The primitive cells used for HLD calculations are presented in panels a. and c., while the extended periodic models used for the AIMD simulations are given in panels b. and d. for $\mathrm{FAPbI}_{3}$ and $\mathrm{MAPbI}_{3}$, respectively.

$\mathrm{MAPbI}_{3}$ using another computational approximation, ie. PBE-D3(BJ) [48 51] (see the SI for a detailed comparison of the results obtained with both PBE-TS and PBED3(BJ)). Since the INS spectrum of MAPI has been extensively analyzed in Ref. [15], we focus here on a detailed interpretation of the INS spectrum of $\mathrm{FAPbI}_{3}$. However, we note that owing to an improved sensitivity of the TOSCA spectrometer, the assignment of a few weak bands in the low-frequency regime has been revised herein. This has been emphasized in Fig. 5, where six $\mathrm{MA}^{+}$librations are now clearly assigned (see the bands labeled as $i-v i)$. Particularly, we note that the highestfrequency external mode (denoted as libration $i$ ) is observed at $18.1 \mathrm{meV}$, whereas the feature at $23 \mathrm{meV}$ is the first overtone of librational mode $v$, largely damped upon mixing. Consequently, the band at ca. $20 \mathrm{meV}$ in the above-discussed difference spectrum does not correspond to $\mathrm{MA}^{+}$vibrations.

By inspection of Fig. 5, one can find an excellent match of the predicted INS spectra of $\mathrm{FAPbI}_{3}$ to the experimental one. Further improvement with the use of AIMD methodology, relying on a larger supercell, points at the importance of a larger number of different local configurations needed to properly describe the spectrum, supporting the finding that the low-temperature structure of $\mathrm{FAPbI}_{3}$ is highly disordered. 28. At this stage, owing to an excellent reproduction of the spectral intensities over the entire frequency range, the assignment of the internal-mode range becomes straightforward, as summarized in Table 1. Despite the use of two different codes (CASTEP vs CP2K), both HLD and low-temperature 
TABLE I: Experimentally observed (INS at $10 \mathrm{~K}$ ) phonon frequencies of $\mathrm{FAPbI}_{3}$ (in meV), as compared to the results of the first-principles calculations (PBE-D3(BJ)). 48 51.

The phonon frequencies are from HLD calculations

(CASTEP), and AIMD simulations (CP2K). Spectral assignments: $\nu$ - stretching; $\delta$ - bending; $\gamma$ - out-of-plane

deformation; Lib. - libration. The mode numbering correspond to those shown in Fig. 5. (Roman and Arabic numerals for the external and internal modes, respectively).

\begin{tabular}{|c|c|c|c|c|}
\hline \multicolumn{4}{|c|}{ Peak Position $[\mathrm{meV}]$} & \multirow{2}{*}{ Assignment } \\
\hline Notation & INS & HLD & MD & \\
\hline 1 & 419.3 & 428.2 & 433.7 & $\nu[\mathrm{N}-\mathrm{H}]$ (antisymmetric) \\
\hline 2 & 406.9 & 416.8 & 417.4 & $\nu[\mathrm{N}-\mathrm{H}]$ (symmetric) \\
\hline 3 & 391.0 & 385.3 & 395.7 & $\nu[\mathrm{C}-\mathrm{H}]$ \\
\hline 4 & 213.9 & 211.0 & 214.0 & $\nu[\mathrm{C}-\mathrm{N}]+\delta\left[\mathrm{NH}_{2}\right]+\delta[\mathrm{CH}]$ \\
\hline 5 & 201.4 & 198.0 & 199.1 & $\delta\left[\mathrm{NH}_{2}\right]($ symmetric $)$ \\
\hline 6 & 188.8 & 190.2 & 190.8 & $\delta\left[\mathrm{NH}_{2}\right]$ (antisymmetric) \\
\hline 7 & 166.7 & 165.4 & 166.9 & $\delta[\mathrm{NCH}]$ \\
\hline 8 & 140.7 & 136.9 & 137.3 & $\delta[\mathrm{CNH}]$ (symmetric) \\
\hline 9 & 125.4 & 124.7 & 124.3 & $\delta[\mathrm{CNH}]+\delta[\mathrm{NCH}]$ \\
\hline 10 & 87.6 & 86.2 & 86.8 & $\gamma\left[\mathrm{NH}_{2}\right]+\gamma[\mathrm{CH}]$ \\
\hline 11 & 74.7 & 75.8 & 74.4 & $\gamma\left[\mathrm{NH}_{2}\right]$ \\
\hline 12 & 72.1 & 72.0 & 72.0 & $\gamma\left[\mathrm{NH}_{2}\right]$ \\
\hline 13 & 67.2 & 67.6 & 68.7 & $\gamma\left[\mathrm{NH}_{2}\right]$ \\
\hline 14 & 63.3 & 61.9 & 62.0 & $\delta[\mathrm{N}-\mathrm{C}-\mathrm{N}]$ \\
\hline$i$ & 19.9 & 22.1 & 24.9 & $\mathrm{FA}^{+}$Lib. (long-axis) \\
\hline$i i$ & 16.1 & 19.6 & 22.7 & $\mathrm{FA}^{+}$Lib. (long-axis) \\
\hline$i i i$ & 13.3 & 14.9 & 16.6 & $\mathrm{FA}^{+}$Lib. (long-axis) \\
\hline$i v$ & 9.3 & 9.5 & 11.4 & $\mathrm{FA}^{+}$Lib. (in-plane) \\
\hline$v$ & 8.1 & 8.3 & 8.8 & FA $^{+}$Lib. (short-axis) \\
\hline$v i$ & 7.2 & 6.9 & 6.1 & FA $^{+}$Lib. (short-axis) \\
\hline$\delta \mathrm{Pb}-\mathrm{I}$ & 3.9 & 3.5 & 3.2 & $\delta[\mathrm{I}-\mathrm{Pb}-\mathrm{I}]+\mathrm{FA}^{+}$Lib. \\
\hline$\delta \mathrm{Pb}-\mathrm{I}$ & 1.4 & 1.6 & 1.7 & $\delta[\mathrm{I}-\mathrm{Pb}-\mathrm{I}]+\mathrm{FA}^{+}$Lib. \\
\hline
\end{tabular}

AIMD simulations provide very similar frequencies. 55 As indicated in Fig. 5, internal and external modes are separated by a large phonon gap of ca. $40 \mathrm{meV}$, which is roughly twice the value found for $\mathrm{MAPbI}_{3}$. At this point, we note that for both parent compounds the lowfrequency part of the spectrum is highly overlapping in terms of energy (ca. 0-20 meV), therefore, being considerably populated at elevated temperatures, where sample mixing is performed. We anticipate that this phenomenon is a key factor making both compounds good candidates for solid solutions.

Because of evident formation of the orientational glass, the analysis of the terahertz part of the INS spectrum of $\mathrm{FAPbI}_{3}$ is far more challenging. Nevertheless, the computational outputs are sufficient to guarantee a general assignment of the respective features. A detailed inspection of the normal modes has allowed to classify the underlying librations into the three main types arising from rotations of an asymmetric top (as displayed as insets to Fig. 5). The normal mode analysis indicates that the range above $15 \mathrm{meV}$ is due to librations along the long molecular axis; the in-plane librations contribute mainly to the most-intense feature at ca. $10 \mathrm{meV}$; whereas the reorientations along the short molecular axis mainly contribute as the low-frequency shoulder bands at ca. 7-8 $\mathrm{meV}$. We note that this idealized picture is disturbed by

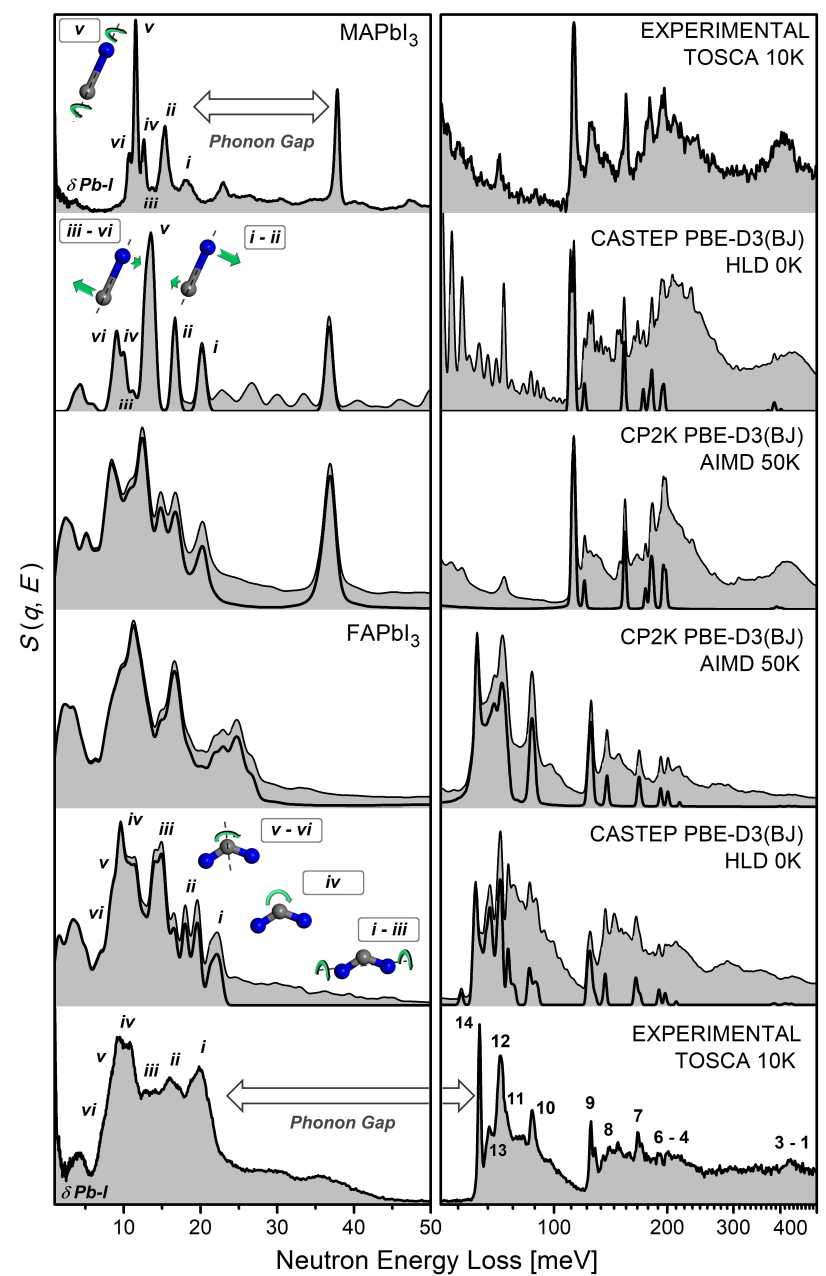

FIG. 5: Experimental (TOSCA $10 \mathrm{~K}$ ) and theoretical (HLD and AIMD) spectra of $\mathrm{FAPbI}_{3}$ (bottom panels) and

$\mathrm{MAPbI}_{3}$ (upper panels) calculated for the structural models presented in Fig. 4 using PBE-D3(BJ) level of theory. 48 54 The contributions from the fundamental transitions in the theoretical spectra are represented by thick lines. Simplified visualization of different types of

librational modes is provided as insets to the figure, with the experimental bands assigned with Roman numerals. For

$\mathrm{FAPbI}_{3}$ the internal modes are further marked with Arabic numerals. See Table 1 for a detailed band assignment.

different local environments and variable strength of the specific forces affecting the $\mathrm{FA}^{+}$cations.

In order to provide more insight into the lowlying features we rely on a detailed decomposition of the hydrogen-projected vibrational density of states (VDOS) into the contribution of each of $48 \mathrm{FA}^{+}$cations included in the extended $\gamma$-phase model. This has been presented in Figure 6, where two extreme cases have been highlighted. The analysis reveals that the upper part of the VDOS is particularly sensitive to the local environment of the $\mathrm{FA}^{+}$cations. The related modes correspond to cation librations about the long molecular axis (see the inset to Fig. 6), giving the strongest 
a.

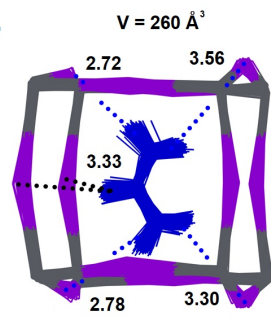

b.
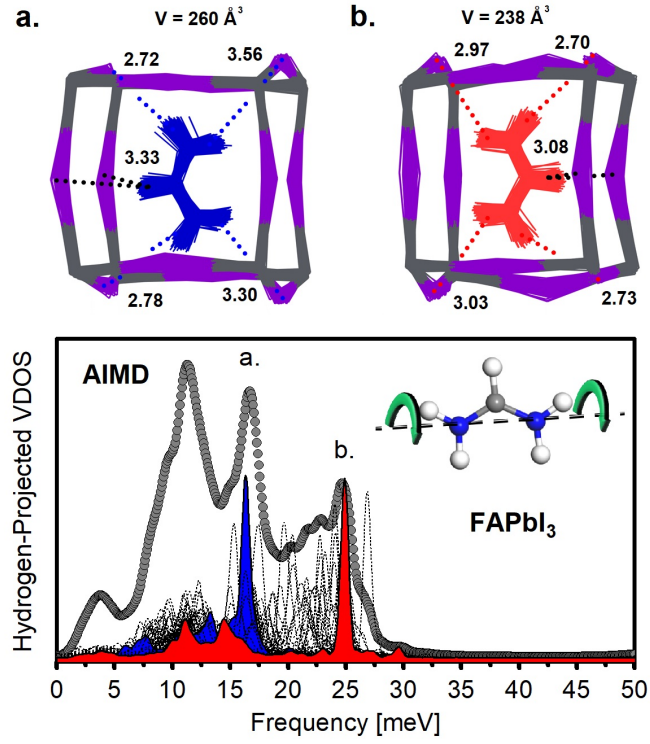

FIG. 6: (bottom panel) Partial hydrogen-projected VDOS spectra calculated for each of $48 \mathrm{FA}^{+}$molecules included in the extended structural model of the $\gamma$-phase of $\mathrm{FAPbI}_{3}$ (see

Fig. 4 b). 56, 57. The most-intense bands in the VDOS spectrum (spread over the range ca. $15-25 \mathrm{meV}$ ) correspond to the molecular libration depicted in the inset. The blue and red shaded curves correspond to two extreme cases of weakly (a.; blue) and strongly (b.; red) H-bonded $\mathrm{FA}^{+}$cations (see the upper panel for time-averaged (30 ps) structures with the same color-coding used). The

time-averaged close-contacts between the hydrogen and iodine atoms are given in Angstroms.

partial contributions to the total intensity due to the largest molecular displacements. As evidenced from the analysis of the time-averaged volumes of the surrounding sub-units along with the $\mathrm{H}-\mathrm{I}$ distances, this effect can be clearly attributed to the strength of the hydrogen-bonds, immobilizing the $\mathrm{FA}^{+}$cations.

Based on the above-given analysis, the INS spectra of the $\mathrm{MA}_{1-\mathrm{x}} \mathrm{FA}_{\mathrm{x}} \mathrm{PbI}_{3}$ mixtures (see Fig. 3 ) can be finally understood. With an increasing amount of the $\mathrm{MA}^{+}$ dopant, the frequency of the lowest-lying internal vibration $(\tau(-\mathrm{C}-\mathrm{N}-))$ undergoes a progressive downward-shift in energy, due to an increasing orientational disorder, further attributed to the weakening of the $\mathrm{MA}^{+}$hydrogen bonding and the disorder-induced expansion of the surrounding cage. Simultaneously, there is an increasing fraction of the $\mathrm{FA}^{+}$cations subjected to stronger hydrogen-bonding interactions with a collapsed inorganic cage, manifested by the upward-shift in energy of the respective highest-energy librational modes. The mechanism is promoted by the extreme softness of the inorganic framework, [36, 37] and a strong overlap of the VDOSs of inorganic [58] and organic counterparts. We note that the INS experiments presented by Ferreira and co-workers 36, 37 link the unstable nature of $\alpha-\mathrm{FAPbI}_{3}$ to its very low bulk modulus and a negative $C_{12}$ elastic constant. Furthermore, the recently proposed transition pathway for the degradation of $\alpha-\mathrm{FAPbI}_{3} \quad 59$ highlights the importance of short-range octahedral distortions. We anticipate that the above-discussed synergistic strengthening of hydrogen-bonding interactions involving the $\mathrm{FA}^{+}$cations results in a locking-effect, which strongly inhibits degradation of the perovskite framework.

\section{CONCLUSIONS}

In conclusion, the combined use of high-resolution INS experiments and advanced $a b$ initio modelling has provided, for the first time, a detailed and quantitative view of the local environment and vibrational dynamics of $\mathrm{FA}^{+}$cations in formamidinium lead-iodide perovskites. The formation of an orientational glass has been confirmed, and a realistic model of the low-temperature structure of $\mathrm{FAPbI}_{3}$ has been proposed and validated by the neutron scattering data. An excellent match between experimental and simulated spectra has been achieved. We have also extended the analysis to heavily substituted solid-state $\mathrm{MA}_{1-\mathrm{x}} \mathrm{FA}_{\mathrm{x}} \mathrm{PbI}_{3}$ mixtures, revealing synergistic dynamics across both $\mathrm{MA}^{+}$and $\mathrm{FA}^{+}$ cations, directly affecting the geometry of the surrounding inorganic framework. Based on these considerations, we have identified important factors contributing to the stabilization of $\mathrm{FAPbI}_{3}$ perovskite structures. We anticipate that these will facilitate the design of efficient routes for the further optimisation of these solid solutions using criteria such as $(i)$ the similarity of the vibrational profiles of the organic guests; (ii) the effective thermal population of low-energy vibrational motions; (iii) or a high molecular mobility of the organic dopants.

\section{EXPERIMENTAL AND COMPUTATIONAL DETAILS}

Sample Preparation: $\mathrm{MA}_{x} \mathrm{FA}_{1-x} \mathrm{PbI}_{3}(x=0.0,0.1,0.4$, and 1.0) powder samples were synthesized using protocols reported elsewhere. [7, 60, 61] The chemical composition and integrity of each sample was further corroborated with ${ }^{1} \mathrm{H}$ NMR following Ref. [7]

Neutron Scattering Experiments: The INS experiments were performed on the TOSCA spectrometer, 41, 45] operating at the ISIS Neutron and Muon Source. The measurements were performed at $T=10 \mathrm{~K}$. Standard corrections were applied to the data within the Mantid software package. 62

Ab Initio Calculations: The modelling of the INS spectra was performed in terms of solid-state formulation of Density Functional Theory (DFT) as implemented in the CASTEP (versions 16.1 and 20.1) 63, 64] and CP2K (version 8.1) codes 65, 66], respectively. The calculations were performed under Periodic Boundary Conditions (PBCs). The PerdewBurke-Ernzerhof (PBE) functional [48] was used all throughout this work. We adopted two different approximations to take into account of van-der-Waals (vdW) interactions in our calculations: Tkatchenko-Scheffler corrections, PBE-TS 67; 
and the D3 correction method of Grimme et al. with BeckeJohnson (BJ) damping, PBE-D3(BJ). 48 51] The phonon eigenvalues and eigenvectors calculated using HLD served as an input for the further modelling of the INS spectra. [52, 53. For the MD simulations, production-run trajectories were used for modelling of the hydrogen-projected VDOS spectra via the explicit calculation of the Velocity AutoCorrelation Function (VACF). The analysis and the post-processing of the MD production runs was made with the help of the TRAVIS code. [56, 57] Alternatively, we have also followed the recently implemented strategy to model the $S(q, E)$ spectra from MD trajectories by means of an approximate treatment of the Debye-Waller factor which accounts for overtone and combination bands as well the specific configuration of the TOSCA spectrometer. 54

More experimental and computational details are provided in the SI.

\section{SUPPLEMENTARY INFORMATION}

This Section includes further details on the INS experiments, ab initio modelling, calculations using simplified mod- els of $\mathrm{FAPbI}_{3}$, analysis of the influence of vdW corrections on the INS spectra, detailed analysis of the low-frequency phonon modes. This material is available free of charge via the Internet at http://pubs.acs.org.

\section{ACKNOWLEDGMENTS}

KD and FFA gratefully acknowledge financial support from the Gipuzkoako Foru Aldundia under Grant Number 2020CIEN-000009-01. This work has been supported by PL-Grid Infrastructure and the PROMETHEUS facility. MK is grateful for financial support from the Swedish Research Council (Grant No. 2016-06258).

KD kindly thanks Maciej Czuchry (PL-Grid) for his continuous technical assistance. KD also gratefully acknowledges Prof. Keith Refson (Royal Holloway, University of London), and Dr. Peter Byrne (University of York) for providing the final version of the CASTEP DFT-D3 implementation and related discussions.
[1] V. Gonzalez-Pedro, E. J. Juarez-Perez, W.-S. Arsyad, E. M. Barea, F. Fabregat-Santiago, I. Mora-Sero and J. Bisquert, Nano Letters, 2014, 14, 888-893.

[2] J. Bisquert and E. J. Juarez-Perez, The Journal of Physical Chemistry Letters, 2019, 10, 5889-5891.

[3] C. Aranda, A. Guerrero and J. Bisquert, ChemPhysChem, 2019, 20, 2587-2599.

[4] F. D. Angelis, ACS Energy Letters, 2019, 4, 853-854.

[5] F. D. Angelis, ACS Energy Letters, 2020, 5, 935-937.

[6] O. J. Weber, B. Charles and M. T. Weller, J. Mater. Chem. A, 2016, 4, 15375-15382.

[7] A. Pisanu, C. Ferrara, P. Quadrelli, G. Guizzetti, M. Patrini, C. Milanese, C. Tealdi and L. Malavasi, J. Phys. Chem. C, 2017, 121, 8746-8751.

[8] Q. Han, S. H. Bae, P. Sun, Y. T. Hsieh, Y. Yang, Y. S. Rim, H. Zhao, Q. Chen, W. Shi, G. Li and Y. Yeng, Adv. Mater., 2016, 28, 2253-2258.

[9] K. Page, J. E. Siewenie, P. Quadrelli and L. Malavasi, Angew. Chem., Int. Ed., 2016, 55, 14320-14324.

[10] A. Bernasconi and L. Malavasi, ACS Energy Lett., 2017, 2, 863-868.

[11] A. Bernasconi, K. Page, Z. Dai, L. Z. Tan, A. M. Rappe and L. Malavasi, J. Phys.Chem. C, 2018, 122, 2826528272.

[12] M. T. Weller, O. J. Weber, P. F. Henry, A. M. Di Pumpo and T. C. Hansen, Chem. Commun., 2015, 51, 41804183.

[13] A. Poglitsch and D. Weber, J. Chem. Phys., 1987, 87, 6373-6378.

[14] T. Baikie, Y. Fang, J. M. Kadro, M. Schreyer, F. Wei, S. G. Mhaisalkar, M. Graetzel and T. J. White, J. Mater. Chem. A, 2013, 1, 5628-5641.

[15] K. Drużbicki, R. S. Pinna, S. Rudić, M. Jura, G. Gorini and F. Fernandez-Alonso, J. Phys. Chem. Lett., 2016, 7, 4701-4709.

[16] J. Breternitz, F. Lehmann, S. A. Barnett, H. Nowell and
S. Schorr, Angew. Chem. Int. Ed., 2019, 59, 424-428.

[17] Y. Rakita, O. Bar-Elli, E. Meirzadeh, H. Kaslasi, Y. Peleg, G. Hodes, I. Lubomirsky, D. Oron, D. Ehre and D. Cahen, Proc. Natl. Acad. Sci., 2017, 114, E5504E5512.

[18] A. Francisco-López, B. Charles, M. I. Alonso, M. Garriga, M. Campoy-Quiles, M. T. Weller and A. R. Goñi, J. Phys.Chem. C, 2020, 124, 3448-3458.

[19] T. Chen, B. J. Foley, C. Park, C. M. Brown, L. W. Harriger, J. Lee, J. Ruff, M. Yoon, J. J. Choi and S.-H. Lee, Sci. Adv., 2016, 2, e1601650.

[20] A. Gold-Parker, P. M. Gehring, J. M. Skelton, I. C. Smith, D. Parshall, J. M. Frost, H. I. Karunadasa, A. Walsh and M. F. Toney, Proc. Natl. Acad. Sci., 2018, 115, 11905-11910.

[21] A. N. Beecher, O. E. Semonin, J. M. Skelton, J. M. Frost, M. W. Terban, H. Zhai, A. Alatas, J. S. Owen, A. Walsh and S. J. L. Billinge, ACS Energy Lett., 2016, 1, 880-887.

[22] D. H. Fabini, T. Hogan, H. A. Evans, C. C. Stoumpos, M. G. Kanatzidis and R. Seshadri, J. Phys. Chem. Lett., 2016, 7, 376-381.

[23] C. C. Stoumpos, C. D. Malliakas and M. G. Kanatzidis, Inorg. Chem., 2013, 52, 9019-9038.

[24] J. J. Choi, L. W. Harriger, J. Ruff, T. Chen, J. Lee, C. Park, C. M. Brown, B. J. Foley, M. Yoon and S.-H. Lee, Sci. Adv., 2016, 2, e1601650.

[25] D. H. Fabini, C. C. Stoumpos, G. Laurita, A. Kaltzoglou, A. G. Kontos, P. Falaras, M. G. Kanatzidis and R. Seshadri, Angew. Chem. Int. Ed., 2016, 55, 15392-15396.

[26] T. Chen, W.-L. Chen, B. J. Foley, J. Lee, J. P. C. Ruff, J. Y. P. Ko, C. M. Brown, L. W. Harriger, D. Zhang, C. Park, M. Yoon, Y.-M. Chang, J. J. Choi and S.-H. Lee, Proceedings of the National Academy of Sciences, 2017, 114, 7519-7524.

[27] S. Sun, Z. Deng, Y. Wu, F. Wei, F. Halis Isikgor, F. Brivio, M. W. Gaultois, J. Ouyang, P. D. Bristowe, 
A. K. Cheetham and G. Kieslich, Chem. Commun., 2017, 53, 7537-7540.

[28] O. J. Weber, D. Ghosh, S. Gaines, P. F. Henry, A. B. Walker, M. S. Islam and M. T. Weller, Chem. Mater., 2018, 30, 3768-3778.

[29] D. H. Fabini, T. A. Siaw, C. C. Stoumpos, G. Laurita, D. Olds, K. Page, J. G. Hu, M. G. Kanatzidis, S. Han and R. Seshadri, J. Am. Chem. Soc., 2017, 139, 1687516884.

[30] J. W. Lee, D. J. Seol, A. N. Cho and N. G. Park, Adv. Mater., 2014, 26, 4991-4998.

[31] Y. Yang, D. P. Ostrowski, R. M. France, K. Zhu, J. Van De Lagemaat, J. M. Luther and M. C. Beard, Nat. Photonics, 2016, 10, 53-59.

[32] N. J. Jeon, J. H. Noh, W. S. Yang, Y. C. Kim, S. Ryu, J. Seo and S. I. Seok, Nature, 2015, 517, 476-480.

[33] T. Jesper Jacobsson, J. P. Correa-Baena, M. Pazoki, M. Saliba, K. Schenk, M. Grätzel and A. Hagfeldt, Energy Environ. Sci., 2016, 9, 1706-1724.

[34] N. P. Gallop, O. Selig, G. Giubertoni, H. J. Bakker, Y. L. A. Rezus, J. M. Frost, T. L. C. Jansen, R. Lovrincic and A. A. Bakulin, The Journal of Physical Chemistry Letters, 2018, 9, 5987-5997.

[35] F. Ambrosio, D. Meggiolaro, E. Mosconi and F. D. Angelis, ACS Energy Letters, 2019, 4, 2013-2020.

[36] A. C. Ferreira, A. Létoublon, S. Paofai, S. Raymond, C. Ecolivet, B. Rufflé, S. Cordier, C. Katan, M. I. Saidaminov, A. A. Zhumekenov, O. M. Bakr, J. Even and P. Bourges, Phys. Rev. Lett., 2018, 121, 1-12.

[37] A. C. Ferreira, S. Paofai, A. Létoublon, J. Ollivier, S. Raymond, B. Hehlen, B. Rufflé, S. Cordier, C. Katan, J. Even and P. Bourges, Commun. Phys., 2020, 3, 1-17.

[38] E. M. Mozur, M. A. Hope, J. C. Trowbridge, D. M. Halat, L. L. Daemen, A. E. Maughan, T. R. Prisk, C. P. Grey and J. R. Neilson, Chem. Mater., 2020, 32, 6266-6277.

[39] F. Cordero, F. Craciun, F. Trequattrini, A. Generosi, B. Paci, A. M. Paoletti and G. Pennesi, J. Phys. Chem. Lett., 2019, 10, 2463-2469.

[40] Z. Chen, H. Zhang, F. Yao, C. Tao, G. Fang and G. Li, Cell Rep., 2020, 1, 100205.

[41] S. F. Parker, F. Fernandez-Alonso, A. J. Ramirez-Cuesta, J. Tomkinson, S. Rudic, R. S. Pinna, G. Gorini and J. F. Castañon, J Phys Conf Ser., 2014, 554, 012003.

[42] R. S. Pinna, S. Rudić, M. J. Capstick, D. J. McPhail, D. E. Pooley, G. D. Howells, G. Gorini and F. FernandezAlonso, Nucl. Instrum. Methods Phys. Res. A, 2017, 870, 79-83.

[43] R. S. Pinna, S. Rudić, S. F. Parker, J. Armstrong, M. Zanetti, G. Škoro, S. P. Waller, D. Zacek, C. A. Smith, M. J. Capstick, D. J. McPhail, D. E. Pooley, G. D. Howells, G. Gorini and F. Fernandez-Alonso, Nucl. Instrum. Methods Phys. Res. A, 2018, 896, 68-74.

[44] R. S. Pinna, M. Zanetti, S. Rudić, S. Parker, J. Armstrong, S. Waller, D. Zacek, C. Smith, S. Harrison, G. Gorini and F. Fernandez-Alonso, J Phys Conf Ser., 2018, 1021, 012029.

[45] M. Zanetti, S. Bellissima, L. del Rosso, F. Masi, M. Chowdhury, A. D. Bonis, L. D. Fresco, C. Scatigno, J. Armstrong, S. Rudić, S. Parker, M. Hartl, D. Colognesi, R. Senesi, C. Andreani, G. Gorini and F. FernandezAlonso, Physica B Condens. Matter., 2019, 562, 107111.

[46] A. Francisco-López, B. Charles, M. I. Alonso, M. Garriga, M. Campoy-Quiles, M. T. Weller and A. R. Goñi,
J. Phys. Chem. C, 2020, 124, 3448-3458.

[47] E. M. Mozur, A. E. Maughan, Y. Cheng, A. Huq, N. Jalarvo, L. L. Daemen and J. R. Neilson, Chem. Mater., 2017, 29, 10168-10177.

[48] J. P. Perdew, K. Burke and M. Ernzerhof, Phys. Rev. Lett., 1996, 77, 3865-3868.

[49] S. Grimme, J. Antony, S. Ehrlich and H. Krieg, J. Chem. Phys., 2010, 132, 154104.

[50] S. Grimme, S. Ehrlich and L. Goerigk, J. Comput. Chem., 2011, 32, 1456-1465.

[51] A. D. Becke and E. R. Johnson, J. Chem. Phys., 2005, 123, 154101.

[52] K. Dymkowski, S. F. Parker, F. Fernandez-Alonso and S. Mukhopadhyay, Physica B Condens. Matter, 2018, 551, 443-448.

[53] Y. Q. Cheng, L. L. Daemen, A. I. Kolesnikov and A. J. Ramirez-Cuesta, J. Chem. Theory Comput., 2019, 15, 1974-1982.

[54] Y. Q. Cheng, A. I. Kolesnikov and A. J. Ramirez-Cuesta, J. Chem. Theory Comput., 2020, 16, 7702-7708.

[55] K. Drużbicki, M. Krzystyniak, D. Hollas, V. Kapil, P. Slavíček, G. Romanelli and F. Fernandez-Alonso, J Phys Conf Ser., 2018, 1055, 012003.

[56] M. Brehm and B. Kirchner, J. Chem. Inf. Model., 2011, 51, 2007-2023.

[57] M. Thomas, M. Brehm, R. Fligg, P. Vöhringer and B. Kirchner, Phys. Chem. Chem. Phys., 2013, 15, 6608.

[58] G. Fisicaro, A. La Magna, A. Alberti, E. Smecca, G. Mannino and I. Deretzis, J. Phys. Chem. Lett., 2020, 11, 1068-1074.

[59] S. Tan, I. Yavuz, M. H. Weber, T. Huang, C.-H. Chen, R. Wang, H.-C. Wang, J. H. Ko, S. Nuryyeva, J. Xue, Y. Zhao, K.-H. Wei, J.-W. Lee and Y. Yang, Joule, 2020, 4, 2426-2442.

[60] A. Mancini, P. Quadrelli, C. Milanese, M. Patrini, G. Guizzetti and L. Malavasi, Inorg. Chem., 2015, 54, 8893-8895.

[61] A. Mancini, P. Quadrelli, G. Amoroso, C. Milanese, M. Boiocchi, A. Sironi, M. Patrini, G. Guizzetti and L. Malavasi, J. Solid State Chem., 2016, 240, 55-60.

[62] O. Arnold, J. C. Bilheux, J. M. Borreguero, A. Buts, S. I. Campbell, L. Chapon, M. Doucet, N. Draper, R. Ferraz Leal, M. A. Gigg, V. E. Lynch, A. Markvardsen, D. J. Mikkelson, R. L. Mikkelson, R. Miller, K. Palmen, P. Parker, G. Passos, T. G. Perring, P. F. Peterson, S. Ren, M. A. Reuter, A. T. Savici, J. W. Taylor, R. J. Taylor, R. Tolchenov, W. Zhou and J. Zikovsky, Nucl. Instrum. Methods Phys. Res. A, 2014, 764, 156-166.

[63] M. C. Payne, M. P. Teter, D. C. Allan, T. Arias and J. D. Joannopoulos, Rev. Mod. Phys., 1992, 64, 1045-1097.

[64] S. J. Clark, M. D. Segall, C. J. Pickard, P. J. Hasnip, M. I. J. Probert, K. Refson and M. C. Payne, Z. Kristallogr., 2005, 220, 567-570.

[65] J. Hutter, M. Iannuzzi, F. Schiffmann and J. VandeVondele, Wiley Interdiscip. Rev. Comput. Mol. Sci., 2013, 4, $15-25$.

[66] J. VandeVondele, M. Krack, F. Mohamed, M. Parrinello, T. Chassaing and J. Hutter, Comput. Phys. Commun., 2005, 167, 103-128.

[67] A. Tkatchenko and M. Scheffler, Phys. Rev. Lett., 2009, 102, 073005-4. 


\title{
SUPPLEMENTARY INFORMATION: Cation dynamics and structural stabilization in formamidinium lead iodide perovskites
}

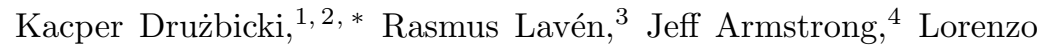 \\ Malavasi, ${ }^{5}$ Felix Fernandez-Alonso, ${ }^{6,7,8,9}$ and Maths Karlsson ${ }^{10}$ \\ ${ }^{1}$ Polish Academy of Sciences, Centre of Molecular and Macromolecular Studies, Sienkiewicza 112, 90-363 Lodz, Poland \\ ${ }^{2}$ Materials Physics Center, CSIC-UPV/EHU, Paseo Manuel de Lardizabal 5, 20018 Donostia - San Sebastian, Spain \\ ${ }^{3}$ Department of Chemistry and Chemical Engineering, \\ Chalmers University of Technology, SE-412 96 Göteborg, Sweden \\ ${ }^{4}$ ISIS Facility, Rutherford Appleton Laboratory, Harwell Oxford, Didcot, Oxfordshire OX11 0QX, U. K. \\ ${ }^{5}$ Department of Chemistry and INSTM, University of Pavia, Viale Taramelli 16, Pavia 27100, Italy \\ ${ }^{6}$ Materials Physics Center, CSIC-UPV/EHU, Paseo Manuel de Lardizabal 5, 20018 Donostia - San Sebastian, Spain \\ ${ }^{7}$ IKERBASQUE, Basque Foundation for Science, Plaza Euskadi 5, 48009 Bilbao, Spain \\ ${ }^{8}$ Donostia International Physics Center (DIPC), \\ Paseo Manuel de Lardizabal 4, 20018 Donostia - San Sebastian, Spain \\ ${ }^{9}$ Department of Physics and Astronomy, University College London, Gower Street, London, WC1E 6BT, U. K. \\ ${ }^{10}$ Department of Chemistry and Chemical Engineering, \\ Chalmers University of Technology, SE-412 96 Göteborg, Sweden
}

\section{INELASTIC NEUTRON SCATTERING}

The INS experiments were performed on the TOSCA spectrometer. [1 5] operating at the ISIS neutron and muon source. Powder samples of $\mathrm{MA}_{x} \mathrm{FA}_{1-x} \mathrm{PbI}_{3}(x$ $=0.0,0.1,0.4$, and 1.0) were loaded into rectangular $\mathrm{Al}$ sample cells which were sealed with In wire. The measurements were performed at $\mathrm{T}=10 \mathrm{~K}$. The pure $\mathrm{FAPbI}_{3}$ sample was measured first as a mixture of the hexagonal and tetragonal polymorphs. A second measurement of $\mathrm{FAPbI}_{3}$ was performed after annealing at $438 \mathrm{~K}$ to form the cubic phase directly prior to the measurements, followed by subsequent quenching to 10 $\mathrm{K}$ where the INS measurements were performed. In this way, the $\mathrm{FAPbI}_{3}$ sample is then in the pure tetragonal phase at $10 \mathrm{~K} .6$, 7 ]

For a powder sample, within the incoherent approximation, the observed neutron-scattering intensity corresponding to the $i$-th vibrational mode at a momentum transfer $q\left[\AA^{-1}\right]$ is given by $[\underline{8}$ :

$$
I\left(q, n E_{i}\right) \propto \frac{\left(q U_{i}\right)^{2 n} \sigma_{\mathrm{inc}}}{n !} \exp \left[-\left(q U_{T o t}\right)^{2}\right]
$$

where $n$ is the quantum number of an $i$-th mode $(n$ $=1$ for a fundamental; $n>1,2,3$ and so forth for overtones and combination bands); $\sigma_{\text {inc }}$ stands for the incoherent scattering cross-section. $U_{i}$ is the mean square atomic displacement in the considered vibration, and the exponential term is known as the Debye-Waller Factor (DWF). $U_{T o t}$ stands for the total root mean square displacement of all the atoms in all (both internal and external) modes, and its magnitude is partially determined by the thermal motion of the molecule. More

\footnotetext{
* E-mail: kacper.druzbicki@ehu.eus Tel: +34943015453
}

rigorous and formal considerations can be found in Ref. [9].

Thus, the INS spectrum of $\mathrm{MA}_{1-\mathrm{x}} \mathrm{FA}_{\mathrm{x}} \mathrm{PbI}_{3}$ will be dominated by contribution from the organic cations, both because of the large incoherent cross section of hydrogen and its low mass relative to other atoms present in the system.

TOSCA is a so-called inverted-geometry time-of-flight spectrometer with two fixed scattering angles. This means that one probes specific trajectories on the $(q, E)$ plane in the forward and backward scattering hemispheres, i.e. with each $E$ corresponding to a specific $q$ value. For TOSCA, this relationship is approximately given by $E\left[\mathrm{~cm}^{-1}\right] \approx 16 q^{2}\left[\AA^{-1}\right]$.

\section{COMPUTATIONAL DETAILS}

The modelling of the INS spectra was performed using both Harmonic Lattice Dynamics (HLD) and Ab Initio Molecular Dynamics (AIMD) under Periodic Boundary Conditions (PBCs). For the HLD calculations we employed the Plane-Wave Pseudo-Potential (PWPP) formulation of Density Functional Theory (DFT) as implemented in the CASTEP code (versions 16.1 and 20.1), 10, 11. The same numerical settings were the same as those used in the previously reported study on $\mathrm{MAPbI}_{3}$. 12 In brief, the Perdew-BurkeErnzerhof (PBE) functional within the GeneralizedGradient-Approximation (GGA) was augmented with semi-empirical dispersion corrections (DFT-D). [13 The core electrons were described by a set of hard, normconserving PPs, while the electronic wave functions were defined using a PW basis set with a kinetic energy cutoff of $900 \mathrm{eV}$. A Monkhorst-Pack grid was used to maintain a constant $k$-spacing of $0.05 \AA^{-1}$. All structures were accurately relaxed to minimize all residual atomic forces. The convergence criteria in the variation of the 
total energy, Hellmann-Feynman forces, external stress (as in the case of full cell-relaxation, unless otherwise stated), maximum displacement, and the self-consistent field (SCF) were defined as $1 \times 10^{-12} \mathrm{eV} /$ atom, $1 \times 10^{-5}$ $\mathrm{eV} / \AA, 0.0001 \mathrm{GPa}, 1 \times 10^{-6} \AA$, and $1 \times 10^{-12} \mathrm{eV} /$ atom, respectively.

We adopted two different approximations to account for van-der-Waals (vdW) interactions in our calculations: Tkatchenko-Scheffler corrections, DFT-vdW(TS) [14; and the D3 correction method of Grimme et al. with Becke-Johnson (BJ) damping, DFT-D3(BJ). 1517. Both DFT-vdW(TS) and DFT-D3(BJ) are atompair-wise DFT-D correction schemes, and we further augmented the latter to include three-body terms.

Following geometry optimization, phonon frequencies and eigenvectors were calculated within the harmonic approximation at the $\Gamma$ point by diagonalizing the massweighted Hessian matrix, $W$, constructed by numerical differentiation of the analytical gradients with respect to atomic displacements. For the finite-difference calculations, we used a displacement amplitude of 0.01 $\AA$. The calculated phonon eigenvalues and eigenvectors served as an input for the further modelling of the INS spectra. [18, 19 .

In the second scheme, we used the CP2K code [20, 21. to perform AIMD simulations on supercell structures (see the main text for more details). These calculations used PBE-D3(BJ) [15 17, 22 in combination with plane-wave (500 Ry) / gaussian atom-centered doublezeta quality basis sets (DZVP-MOLOPT) along with Goedecker-Teter-Hutter (GTH) pseudopotentials. Convergence criteria were chosen to be consistent with the accuracy of the parallel CASTEP calculations. This approach provides a similar level of accuracy for both computational codes 23. Such consistetncy has been further verified for $\mathrm{MAPbI}_{3}$ via comparison with earlier published results (see Section IV) [12]. A set of classically-thermostatted (NVT; Nosé-Hoover) timeevolved simulations was performed in the framework of Born-Oppenheimer Molecular Dynamics (BOMD). In each case, 20-ps simulations at $150 \mathrm{~K}$ were followed by a 10-ps equilibration at $50 \mathrm{~K}$, and a subsequent 30-ps production run. Using 576 processors at the Polish highperformance computing facility PROMETHEUS, 24] these calculations produced about 2.5 ps per day.

For the MD simulations, production-run trajectories served as input for the modelling of the hydrogenprojected Vibrational Densities Of States (VDOS) via the explicit calculation of the Velocity AutoCorrelation Function (VACF), for direct comparison with INS data. The VDOS for a particular atom $j$ is given by the Fouriertransform of the VACF according to:

$$
V D O S=\sum_{j}^{N} \int_{0}^{\infty} \frac{<\nu(t)_{j} \nu(0)_{j}>}{<\nu(0)_{j}^{2}>} \exp (-i \omega t) d t,
$$

where $\nu(t)_{j}$ is the velocity at time $t$. The analysis and the post-processing of the BOMD production runs was made with the help of the TRAVIS code. [25, 26] Alternatively, we have also followed the recently implemented strategy to model the INS spectra from MD trajectories by means of an approximate treatment of the Debye-Waller factor which accounts for overtone and combination bands as well the specific configuration of the TOSCA spectrometer. 27.

\section{THE SIMPLIFIED MODELS OF THE TETRAGONAL PHASE OF FAPBI}

Using the structure of the $\beta$-tetragonal phase of $\mathrm{FAPbI}_{3}$ reported by Weber et al. [7] as starting point, we found that it becomes necessary to consider a range of $\mathrm{FA}^{+}$configurations within the unit cell. The transformation of the initial $P 4 / \mathrm{mbm}$ structure into the alternative ones results in a lowered global symmetry. Following these considerations, we have examined a number of alternative models, with the internal coordinates constrained by symmetry in each case. These have been illustrated in Fig. S1. (see models a.-f.). Although a larger number of different configurations was tested, the discussed ones were selected as giving visibly distinct INS spectra.

(a.) The structure was simply derived from the initial $P 4 / m b m$ model, with only one $\mathrm{FA}^{+}$molecule per asymmetric unit. This model has $P \overline{4} 2_{1} m$ symmetry, based on the cell constants fixed to the experimental values $(200 \mathrm{~K})$. 7

(b.) The model was built by running simple BOMD simulations with CASTEP (NVT at 200K), where the coordinates of the inorganic cage were fixed, and the preferred orientation of $\mathrm{FA}^{+}$cation was found. The resulting structure was further symmetrized to the closest, $P c$ point group, and successively fully optimized at atmospheric pressure.

(c.) This is a $1 \times 1 \times 2$ supercell of $(a$.$) , with the \mathrm{FA}^{+}$ cations reoriented by $180^{\circ}$ in the $c$-direction, and by $90^{\circ}$ in the $a b$-plane, respectively. This model consists of two cations per asymmetric unit, preserving the $P \overline{4} 2_{1} m$ symmetry. The model was obtained based on the cell constants fixed to the experimental values. [7]

$(d$.$) This is a variation of model (b$.$) above. It$ consists of one molecule per asymmetric unit, where the symmetry operators of the $P 2_{1}$ space group transform the cations by $180^{\circ}$ in the $a$-direction. The structure was further fully optimized at atmospheric pressure.

(e.) This is a variation of the $2 \times 1 \times 1$ supercell in model $(d$.$) , with two \mathrm{FA}^{+}$cations per asymmetric unit, thereby preserving $P 2_{1}$ symmetry. The structure was also fully optimized at atmospheric pressure.

$(f$.$) Finally, the structures is an analogue of model (e.),$ containing four, fully independent $\mathrm{FA}^{+}$cations (global symmetry $P 1$ ), being fully optimized at the atmospheric pressure without any symmetry constraints.

The INS spectra predicted with HLD for each structure 
a.

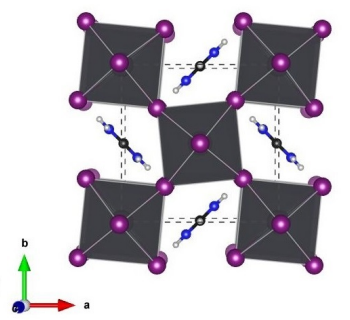

C.

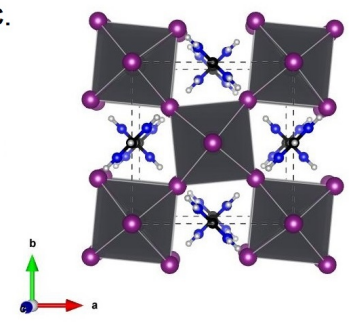

e.

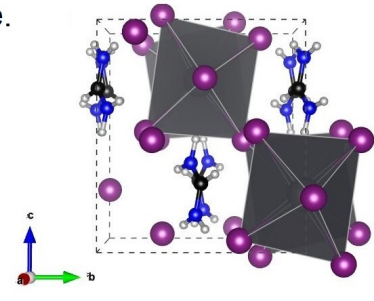

b.

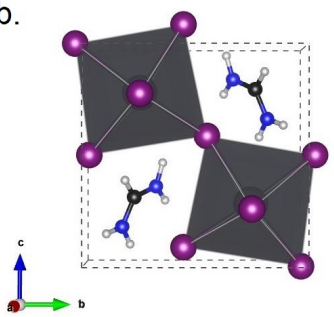

d.

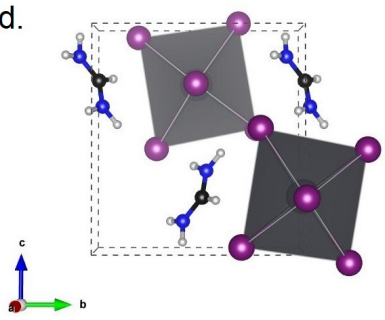

f.

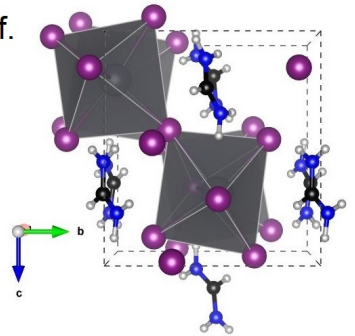

FIG. 1: Alternative, simple models of the tetragonal phase of $\mathrm{FAPbI}_{3}$, obtained with CASTEP/PBE-TS calculations. See the main text for a detailed description. Note a different convention of the cell axes adopted in each case.

considered are presented in Fig. 2. These have been confronted with the experimental spectra recorded on TOSCA at $10 \mathrm{~K}$. By inspection of the figure one can note that the INS spectrum of $\mathrm{FAPbI}_{3}$ is highly sensitive to the cation orientation, particularly in the range below $400 \mathrm{~cm}^{-1}$.

The local arrangement of the $\mathrm{FA}^{+}$cations affects the high-frequency regime mainly in terms of the resulting transition intensities and the presence of the shoulder bands all across $500-1200 \mathrm{~cm}^{-1}$. This range can be attributed to purely internal $\mathrm{FA}^{+}$vibrations.

While each model facilitates a qualitative interpretation of the spectra above $400 \mathrm{~cm}^{-1}$, none of them provides an acceptable reproduction of the low-frequency regime observed experimentally. This range can be attributed to external vibrations of the $\mathrm{FA}^{+}$ions, where the rotational and translational degrees of freedom are transformed to a $\mathrm{PbI}_{3}$-mixed lattice phonons. Such a discrepancy has been confirmed by Mozur et al., with the DFT calculations performed for an analogous $\mathrm{FAPbBr}_{3}$ system, using a simplified model of the tetragonal phase. 28.

Closer inspection of the external modes in the a. and b. models also allows to exclude the presence of a highly ordered crystal structure with the cations

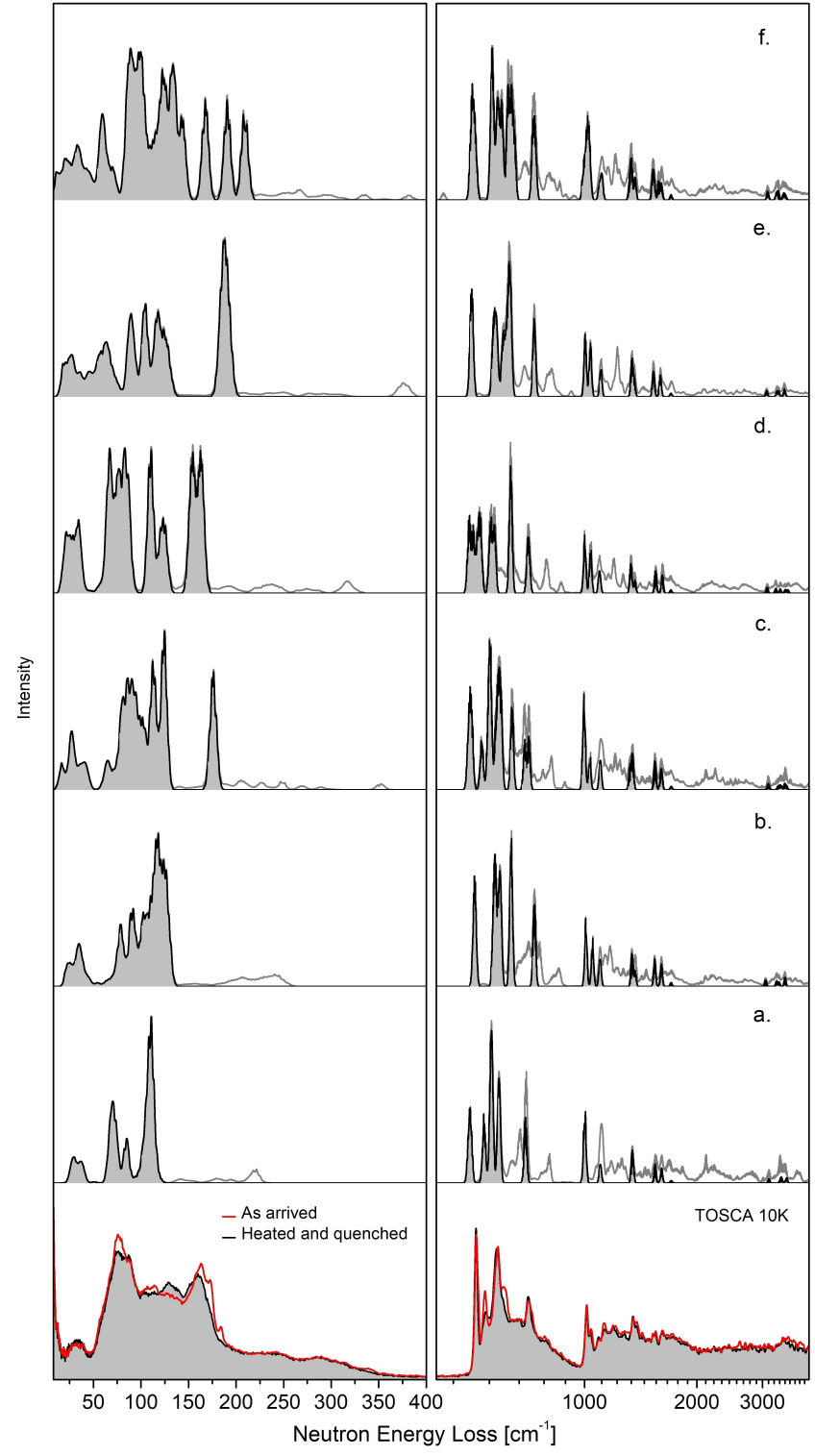

FIG. 2: Comparison of the experimental (TOSCA 10K) and theoretical INS spectra of $\mathrm{FAPbI}_{3}$. The theoretical spectra correspond to the (a. - f.) models presented in Fig. S1. The calculations were performed with pair-wise vdW corrections to PBE (PBE-TS). The energy scale is given in inverse centimeters.

oriented preferentially along a unique direction. While extending the number of the nonequivalent $\mathrm{FA}^{+}$cations, and so accounting for orientational disorder, we note a progression of the low-part of the VDOS toward higher wavenumbers.

We conclude that the simplified models of the tetragonal phase, derived from the model of an intermediate-temperature $\beta$-phase $(P 4 / \mathrm{mbm}), \quad$ are insufficient to describe the INS response of $\mathrm{FAPbI}_{3}$ in the low-temperature regime. For the low-temperature limit, one should consider the system as an orientational glass, for which no simple definition of local order appears to be possible. These results are in line with 
the diffraction studies presented by Weber et al. [7] and supports the dielectric spectroscopy reported by Fabini et al. 29] In order to improve the agreement with the experimental data, we have employed alternative, more extended models of the low-temperature phase of $\mathrm{MAPbI}_{3}$ presented in the main text.

\section{THE INFLUENCE OF THE VDW CORRECTIONS ON THE INS SPECTRA}

The consistency of the HLD outputs obtained by means of the two different schemes used to correct for missing vdW energy contributions to the PBE functional has been examined by confronting the computational results with the experimental spectra measured on TOSCA for both $\mathrm{MAPbI}_{3}$ and $\mathrm{FAPbI}_{3}$. These results are collected in Figs. S3 - S5, respectively. The results were obtained through the calculations performed for the primitive cells described in the main text, where the unit cells of $\mathrm{MAPbI}_{3}$ were fully-relaxed under atmospheric pressure, contrary to $\mathrm{FAPbI}_{3}$, for which the cell constants were fixed to the values measured at $15 \mathrm{~K}$ by Chen et al. 6]

The excellent correlation between the phonon frequencies calculated with both approaches (Fig. S3) justify the use of both computational strategies for the computational modeling of the title systems. While providing nearly identical treatment of the vibrational properties, some deviations appear in the range below $30 \mathrm{meV}$, where the vibrational dynamics of the organic cations is largely dictated by the vibrations of inorganic framework due to the coupling of both organic and inorganic counterparts. This has been presented in more detail in Fig. S4.

By referring to Fig. S4 and the intensity distributions provided therein, we note that both approaches provide a satisfying match to the experimental data. Some differences in the low-frequency region of the $\mathrm{FAPbI}_{3}$ spectrum can be attributed to artificial stress present in the fixed-cell calculations, 6] where both PBE-D3(BJ) and PBE-TS structures are slightly off their equilibrium volumes at $0 \mathrm{~K}$.

\section{POTENTIAL ENERGY DISTRIBUTION ANALYSIS OF THE PHONON MODES OF $\begin{array}{lll}\mathrm{FAPBI}_{3} & \mathrm{AND} \mathrm{MAPBI}_{3}\end{array}$}

To support our discussion in the main text, Fig. S5 gives a detailed analysis of the potential energy distribution for each normal mode of vibration predicted by both PBE-D3(BJ) and PBE-TS. This decomposition focuses on the low-energy motions of $\mathrm{FAPbI}_{3}$ and $\mathrm{MAPbI}_{3}$. We note that the vibrations of the inorganic cage largely dominate the potential energy landscape in the range below $8 \mathrm{meV}$. Librations predicted at ca. 8-16 meV also contain considerable contributions from the motions of the centre-of-mass of the organic cations (Transl.), therefore evincing strong couplings to the deformation of the inorganic framework.

At this point, we also address the question how the mass of the cation affects the terahertz range of vibrations in both compounds considered. This can be understood by focusing on the translational contributions. From comparison of the stacked histograms presented for $\mathrm{MAPbI}_{3}$ and $\mathrm{FAPbI}_{3}$, it appears that the shift is at the order of ca. $2 \mathrm{meV}$. This can be illustrated by a slight change in energy of the two upper-frequency phonon modes with dominating translational character, i.e. ca. 20 and $18 \mathrm{meV}$ for $\mathrm{MAPbI}_{3}$, and $\mathrm{FAPbI}_{3}$, respectively. Further insight is, however, hindered by a highly-mixed nature of the modes.
[1] S. F. Parker, F. Fernandez-Alonso, A. J. Ramirez-Cuesta, J. Tomkinson, S. Rudic, R. S. Pinna, G. Gorini and J. F. Castañon, Journal of Physics: Conference Series, 2014, 554, 012003.

[2] R. S. Pinna, S. Rudić, M. J. Capstick, D. J. McPhail, D. E. Pooley, G. D. Howells, G. Gorini and F. Fernandez-Alonso, Nuclear Instruments and Methods in Physics Research Section A: Accelerators, Spectrometers, Detectors and Associated Equipment, 2017, 870, 79-83.

[3] R. S. Pinna, S. Rudić, S. F. Parker, J. Armstrong, M. Zanetti, G. Škoro, S. P. Waller, D. Zacek, C. A. Smith, M. J. Capstick, D. J. McPhail, D. E. Pooley, G. D. Howells, G. Gorini and F. Fernandez-Alonso, Nuclear Instruments and Methods in Physics Research Section
A: Accelerators, Spectrometers, Detectors and Associated Equipment, 2018, 896, 68-74.

[4] R. S. Pinna, M. Zanetti, S. Rudić, S. Parker, J. Armstrong, S. Waller, D. Zacek, C. Smith, S. Harrison, G. Gorini and F. Fernandez-Alonso, Journal of Physics: Conference Series, 2018, 1021, 012029.

[5] M. Zanetti, S. Bellissima, L. del Rosso, F. Masi, M. Chowdhury, A. D. Bonis, L. D. Fresco, C. Scatigno, J. Armstrong, S. Rudić, S. Parker, M. Hartl, D. Colognesi, R. Senesi, C. Andreani, G. Gorini and F. Fernandez-Alonso, Physica B: Condensed Matter, 2019, 562, 107-111.

[6] T. Chen, W. L. Chen, B. J. Foley, J. Lee, J. P. Ruff, J. Y. Ko, C. M. Brown, L. W. Harriger, D. Zhang, C. Park, M. Yoon, Y. M. Chang, J. J. Choi and S. H. Lee, Proc. 

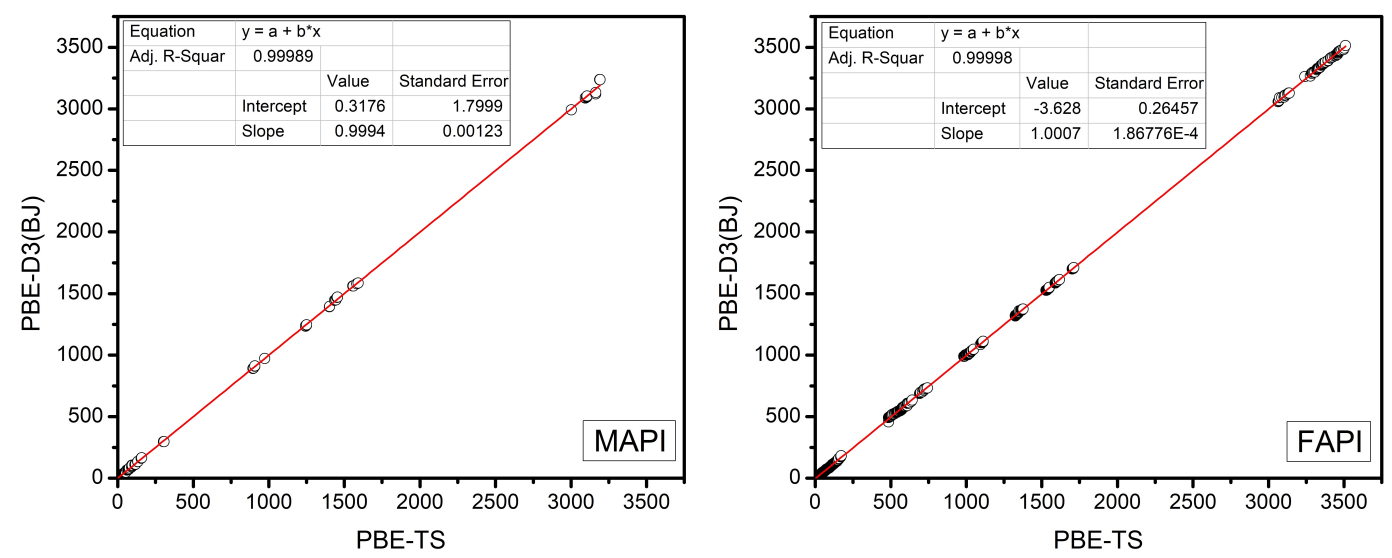

FIG. 3: Correlation between the phonon frequencies (in inverse centimeters) for the low-temperature phases of (left) $\mathrm{MAPbI}_{3}$, and $\mathrm{FAPbI}_{3}$, as calculated by means of different vdW corrections to PBE functional (Grimme's D3 with Becke-Johnson Damping, PBE-D3(BJ), and Tkatchenko-Scheffler, PBE-TS.)

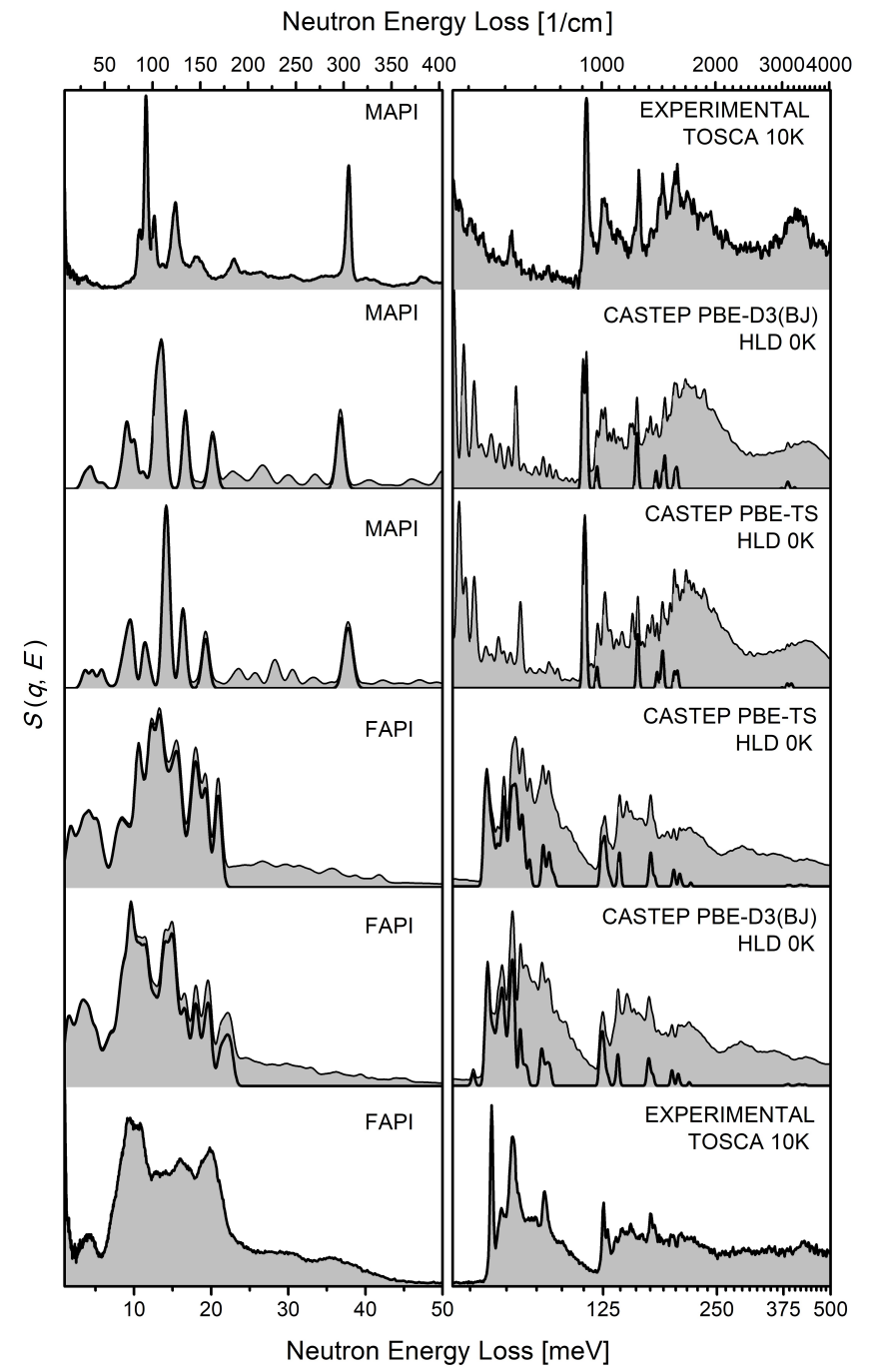

FIG. 4: Comparison of the experimental (TOSCA 10K) and theoretical INS spectra of $\mathrm{MAPbI}_{3}$ and FAPbI 3 calculated with PBE-D, using pair-wise (TS) and triple-wise (D3(BJ)) vdW corrections, respectively. 

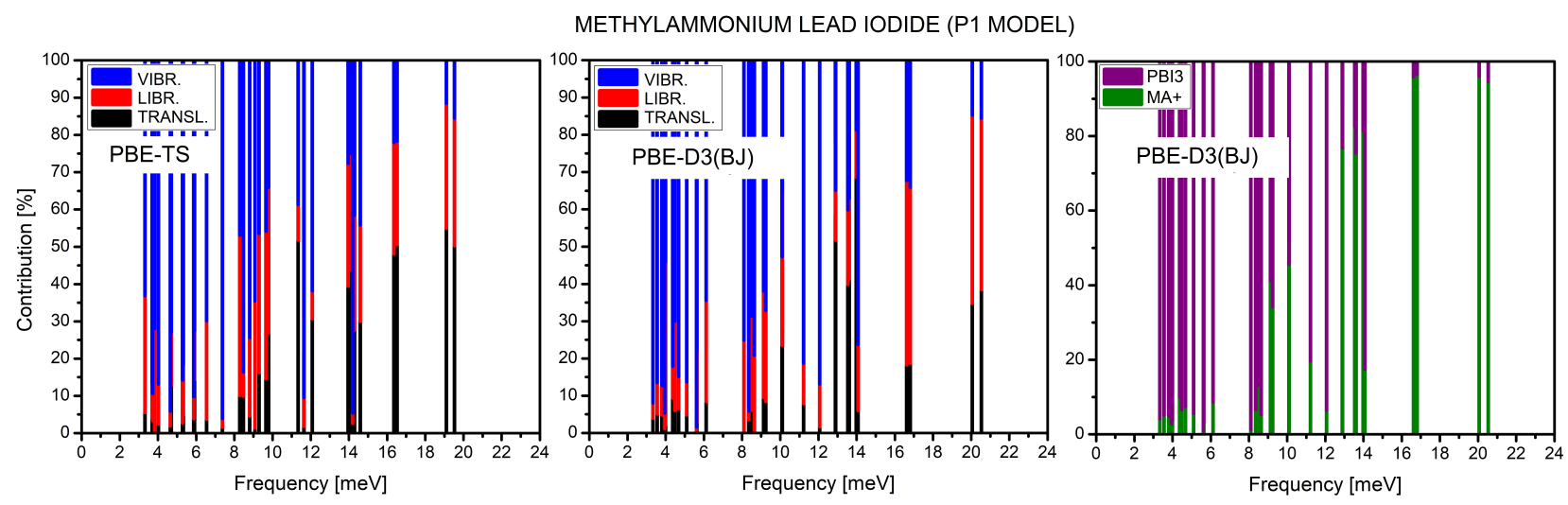

FORMAMIDINIUM LEAD IODIDE (P1 MODEL, GAMMA PHASE)
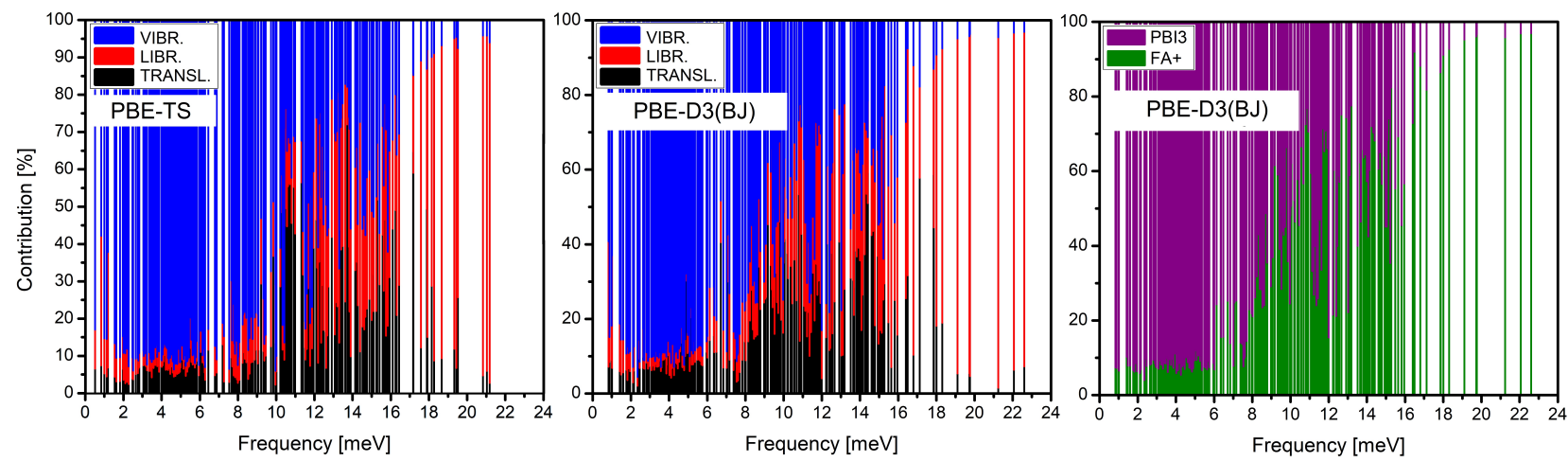

FIG. 5: Stacked histograms showing the energy decomposition of the low-frequency phonon modes of $\mathrm{MAPbI}_{3}$ (upper panels) and

$\mathrm{FAPbI}_{3}$ (bottom panels), as calculated for their low-temperature phases by means of PBE-TS and PBE-D3(BJ). The percentage contributions arise from the translations (TRANSL.) and librations (LIBR.) and the internal molecular vibrations. See Ref. 30. for a detailed description of the numerical procedure applied for the decomposition of the dynamic matrix. The phonon frequencies are given in $\mathrm{meV}$, in line with the data presented in the main text.

Walker, M. S. Islam and M. T. Weller, Chem. Mater., 2018, 30, 3768-3778.

[8] P. C. H. Mitchell, S. F. Parker, A. J. Ramirez-Cuesta and J. Tomkinson, Vibrational Spectroscopy with Neutrons, WORLD SCIENTIFIC, 2005.

[9] D. L. Price and F. Fernandez-Alonso, in Experimental Methods in the Physical Sciences, Elsevier, 2013, pp. 1136.

[10] M. C. Payne, M. P. Teter, D. C. Allan, T. Arias and J. D. Joannopoulos, Rev. Mod. Phys., 1992, 64, 1045-1097.

[11] S. J. Clark, M. D. Segall, C. J. Pickard, P. J. Hasnip, M. I. J. Probert, K. Refson and M. C. Payne, $Z$. Kristallogr., 2005, 220, 567-570.

[12] K. Druzbicki, R. S. Pinna, S. Rudić, M. Jura, G. Gorini and F. Fernandez-Alonso, J. Phys. Chem. Lett., 2016, 7, 4701-4709.

[13] E. McNellis, J. Meyer and K. Reuter, Phys. Rev. B, 2009, 80, 205414-10.

[14] A. Tkatchenko and M. Scheffler, Phys. Rev. Lett., 2009, 102, 073005-4.

[15] S. Grimme, J. Antony, S. Ehrlich and H. Krieg, The Journal of Chemical Physics, 2010, 132, 154104.

[16] S. Grimme, S. Ehrlich and L. Goerigk, Journal of Computational Chemistry, 2011, 32, 1456-1465.

[17] A. D. Becke and E. R. Johnson, The Journal of Chemical Physics, 2005, 123, 154101.
[18] K. Dymkowski, S. F. Parker, F. Fernandez-Alonso and S. Mukhopadhyay, Physica B: Condensed Matter, 2018, 551, 443-448.

[19] Y. Q. Cheng, L. L. Daemen, A. I. Kolesnikov and A. J. Ramirez-Cuesta, Journal of Chemical Theory and Computation, 2019, 15, 1974-1982.

[20] J. Hutter, M. Iannuzzi, F. Schiffmann and J. VandeVondele, Wiley Interdisciplinary Reviews: Computational Molecular Science, 2013, 4, 15-25.

[21] J. VandeVondele, M. Krack, F. Mohamed, M. Parrinello, T. Chassaing and J. Hutter, Computer Physics Communications, 2005, 167, 103-128.

[22] J. P. Perdew, K. Burke and M. Ernzerhof, Phys. Rev. Lett., 1996, 77, 3865-3868.

[23] K. Drużbicki, M. Krzystyniak, D. Hollas, V. Kapil, P. Slavíček, G. Romanelli and F. Fernandez-Alonso, Journal of Physics: Conference Series, 2018, 1055, 012003.

[24] plgrid.pl/en, accessed Feb 03 2021,.

[25] M. Brehm and B. Kirchner, Journal of Chemical Information and Modeling, 2011, 51, 2007-2023.

[26] M. Thomas, M. Brehm, R. Fligg, P. Vöhringer and B. Kirchner, Physical Chemistry Chemical Physics, 2013, 15, 6608 .

[27] Y. Q. Cheng, A. I. Kolesnikov and A. J. Ramirez-Cuesta, Journal of Chemical Theory and Computation, 2020, 16, 
$7702-7708$.

[28] E. M. Mozur, M. A. Hope, J. C. Trowbridge, D. M. Halat, L. L. Daemen, A. E. Maughan, T. R. Prisk, C. P. Grey and J. R. Neilson, Chem. Mater., 2020, 32, 6266-6277.

[29] D. H. Fabini, T. Hogan, H. A. Evans, C. C. Stoumpos,
M. G. Kanatzidis and R. Seshadri, The Journal of Physical Chemistry Letters, 2016, 7, 376-381.

[30] A. D. Burnett, J. Kendrick, J. E. Cunningham, M. D. Hargreaves, T. Munshi, H. G. M. Edwards, E. H. Linfield and A. G. Davies, ChemPhysChem, 2010, 11, 368-378. 


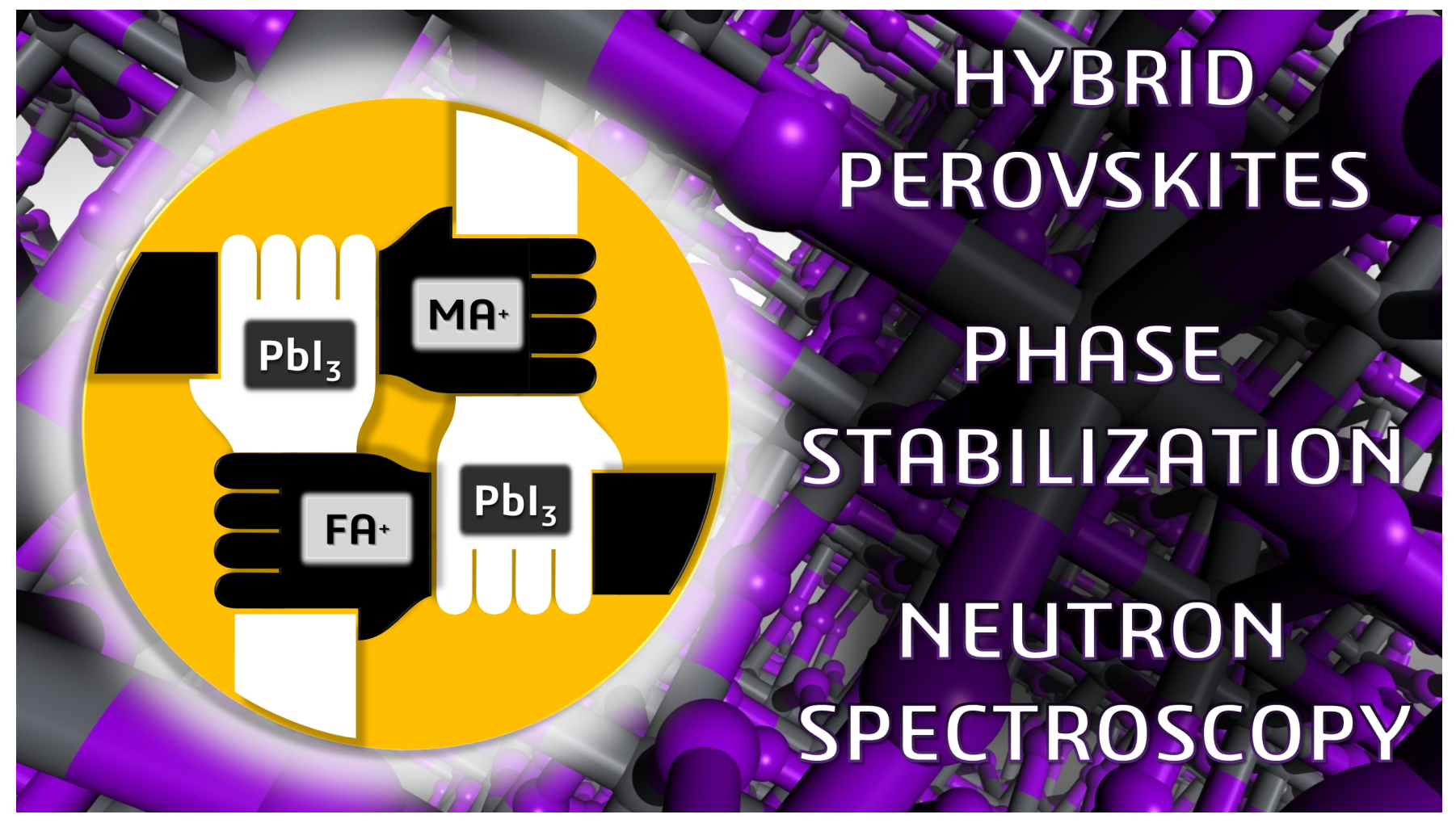

FIG. 7: GRAPHICAL ABSTRACT 